\title{
ARNTL2, an Immunoregulation-Related Prognostic Biomarker in Pan-Cancer and Lung Adenocarcinoma
}

Gujie Wu ( $2013320130 @ s t m a i l . n t u . e d u . c n$ )

Nantong University Affiliated Hospital: Affiliated Hospital of Nantong University https://orcid.org/00000003-3555-0988

\section{Wenmiao Wang}

Nantong University

Zheng Yang

Nantong University

Qun Xue

Nantong University

\section{Primary research}

Keywords: lung adenocarcinoma, prognostic biomarker, macrophages, immunosuppressive microenvironment, anti-cancer drug

Posted Date: November 23rd, 2021

DOI: https://doi.org/10.21203/rs.3.rs-1065822/v1

License: (c) (1) This work is licensed under a Creative Commons Attribution 4.0 International License. Read Full License 


\section{Abstract \\ Background}

ARNTL2 is a member of the PAS superfamily that promotes tumor progression. However, the role of ARNTL2 in lung adenocarcinoma (LUAD) remains unclear. The purpose of our study was to investigate the function of ARNTL2 in LUAD.

\section{Methods}

The expression, clinical features, and prognostic role of ARNTL2 in pan-cancer were evaluated using The Cancer Genome Atlas and Genotype-Tissue Expression data. GSEA and GSVA of ARNTL2 were performed using the R package "clusterProfiler." The correlation between immune cell infiltration level and ARNTL2 expression was analyzed using two sources of immune cell infiltration data, including the TIMER2 and ImmuCellAI database. Finally,we analyzed the correlation between ARNTL2 and IC50 of 192 drugs.

\section{Results}

ARNTL2 was substantially overexpressed in LUAD and pan-cancer. High ARNTL2 expression predicted poor survival in patients with LUAD. We also found that ARNTL2 expression was positively associated with the infiltration levels of immunosuppressive cells, such as tumor associated macrophages, cancer associated fibroblasts and Tregs. Among the 192 anti-cancer drugs, ARNTL2 expression was positively correlated with IC50 of 114 anti-cancer drugs, such as SB505124, Doramapimod, Nutlin-3a (-), Sabutoclax, AZD5991, PF-4708671, Elephantin, PRIMA-1MET, Sorafenib, Vorinostat, and MK-2206.

\section{Conclusions}

Our results revealed that ARNTL2 is a potential prognostic biomarker in LUAD. An elevated ARNTL2 expression indicates an immunosuppressive microenvironment, and targeted therapies against ARNTL2 have excellent potential.

\section{Introduction}

Although huge progress has been made in the treatment of lung adenocarcinoma (LUAD) in recent years, the prognosis of LUAD patients is still very low, mainly because most patients are diagnosed in the final stage and lack of effective specific therapeutic targets ${ }^{[1-3]}$. Immunotherapy has been shown to have advantages for LUAD, but most patients are still not sensitive to immunotherapy ${ }^{[4,5]}$. Therefore, it is urgent to explore the underlying mechanism of LUAD and determine the key biomarkers and potential targets for LUAD patients. 
Recent studies have showed the evidence that the tumor microenvironment (TME) plays an important role in the development of LUAD, and weakens the response of LUAD patients to treatment ${ }^{[6]}$. TIME in the TME were remodeled and lost their original function by tumor cells. For example, tumor-associated macrophages (TAMs), especially M2-like TAMs, were remolded directly or indirectly by tumor cells and in turn played an immunosuppressive and tumor-promoting role ${ }^{[7-10]}$. However, only a few genes form part of the routine prognostic markers in LUAD, but TIME-derived biomarker genes are potential additions to existing predictive and prognostic marker panels.

ARNTL2 is one of the PAS superfamily proteins. It was recently reported that ARNTL2 could promote the progression of pancreatic ductal adenocarcinoma by TGF/BETA pathway ${ }^{[11]}$. Another study showed that the deletion of ARNTL2 could suppress colon carcinoma by inactivating PI3K/AKT pathway ${ }^{[12]}$. In clear cell renal cell carcinoma, ARNTL2 is involved in poor survival and immune infiltration ${ }^{[13]}$. However, the study of ARNTL2 in most tumor types and LUAD is insufficient.

In our study, we comprehensively analyzed the role of ARNTL2 using multi-omics data from TCGA database for 33 cancers, including expression, clinical features, prognostic roles, DNA methylation, copy number alteration (CNA), and mutation status of ARNTL2. The correlation between ARNTL2 expression and infiltration level of immune cells were further assessed. The high expression of ARNTL2 predicted the immunosuppressive TIME, which may lead to poorer survival of tumor patients with a high ARNTL2 expression. Thus, targeting ARNTL2 may be a potential cancer therapy method.

\section{Materials And Methods}

\section{Data source}

The RNAseq data and clinical information of The Cancer Genome Atlas (TCGA) and Genotype-Tissue Expression (GTEx) were downloaded from the UCSC Xena (https://xenabrowser.net/datapages/) database. The immune cell infiltration data of TCGA were downloaded from a TIMER2 (http://timer.cistrome.org/) and ImmuCellAl (http://bioinfo.life.hust.edu.cn/ImmuCellAl\#!/) database. The methylation and CNA of ARNTL2 were downloaded from the cBioPortal database (https://www.cbioportal.org/). The IC50 values of 192 drugs and RNAseq data for a total of 809 cell lines were downloaded from GDSC (https://www.cancerrxgene.org/).

\section{GSEA and GSVA}

The correlation analysis between ARNTL2 expression level and all genes was conducted in each tumor types, and Pearson's correlation coefficient was calculated. The genes correlated with ARNTL2 $(p<0.05)$ were ranked and subjected to GSEA analysis. Gene Ontology (GO), Kyoto Encyclopedia of Genes and Genomes (KEGG) and Reactome database were used for GSEA using the R package "clusterProfiler." For the GSVA, the hallmark pathways were downloaded from MSigDB database. The GSVA was conducted by the R package "GSVA" using "ssGSEA" algorithm. 


\section{Immune infiltration analysis}

The R package "ESTIMATE" was used to assess the stromal and immune score of each patient in TCGA cohort. Two different methods were employed to evaluate the association between ARNTL2 and immune cell infiltration. For the first method, the immune cell infiltration data was downloaded from TIMER2 database and was used to explore the association between ARNTL2 and immune cell infiltration. For the second method, we obtained the infiltration data of 24 immune cells from ImmuCellAl database and conducted the correlation analysis between ARNTL2 expression and infiltration level of 24 immune cells. In addition, the correlation between immunoregulation-related genes and ARNTL2 was analyzed in TCGA pan-cancer.

\section{Results}

\section{Expression of ARNTL2 in pan-cancer}

We first assessed the expression of ARNTL2 using TCGA and GTEx data. We revealed that ARNTL2 was over-expressed in 26 among 33 cancers types, including BLCA, BRCA, CESC, CHOL, COAD, DLBC, ESCA, GBM, HNSC, KIRC, KIRP, LAML, LGG, LIHC, LUAD, LUSC, OV, PAAD, PCPG, READ, STAD, TGCT, THCA, THYM, UCEC, and UCS. In addition, ARNTL2 was low-expressed in only three cancer type, including ACC, PRAD, and SKCM (Figure 1A). To compare the expression of ARNTL2 only in tumor tissues, we found that ARNTL2 was highest expressed in HNSC and lowest in UVM (Figure 1B). For normal tissues from the GTEx database, results revealed that the expression of ARNTL2 was highest in vagina tissues and lowest in muscle (Figure 1C).

Regarding the tumor and adjacent normal tissues in TCGA cohort, ARNTL2 was also observed to be overexpressed in 15 cancers, such as BLCA, CESC, CHOL, COAD, ESCA, HNSC, KIRC, KIRP, LIHC, LUAD, LUSC, PCPG, READ, STAD, and THCA. (Figure 2A-O), while lowly expressed in KICH (Figure 2P).

We further investigated ARNTL2 expression at various tumor stages. The results revealed that ARNTL2 expression was elevated in the relative worse tumor stages in ACC, BLCA, LUAD, PAAD, UCEC, and UVM (Figure 3A-F).

\section{Gene alteration of ARNTL2}

Gene mutation status, DNA methylation, and CNA are main factors that influence gene expression. Thus, we assessed the mutation status, DNA methylation, and CNA of ARNTL2. We found that the highest alteration frequency of ARNTL2 in NSCLC patients was "Amplification". For the correlation between ARNTL2 and CNA, we revealed that ARNTL2 expression were positively correlated with CNA in most tumor types, including LUAD ( $r=0.41$ ) (Figure 4B). In addition, we also found that the promoter methylation level of ARNTL2 was negatively correlated with ARNTL2 expression in LUAD $(r=-0.59)$, which may also induce high expression of ARNTL2 (Figure 4C).

\section{Prognostic role of ARNTL2}


To assess the prognostic role of ARNTL2, the univariate Cox regression analysis (UniCox) and KaplanMeier survival analysis was performed. Results of the UniCox indicated that ARNTL2 was a risk factor for overall survival (OS), disease-free interval (DFI), progression-free interval (PFI), and disease-specific survival (DSS) of LUAD and PAAD patients (Figure 5A-D). Kaplan-Meier OS analysis proved that an elevated ARNTL2 expression predicted worse OS of patients with LGG, LUAD, MESO, PAAD, UCEC, and UVM (Figure 6A-F).

\section{GSEA and GSVA of ARNTL2}

To explore the potential pathways ARNTL2 may involve in tumor progression, we further conducted GSEA of ARNTL2. The top 50 genes most positively and negatively correlated with ARNTL2 were showed (Figure 7A-B). For the results of GSEA-GO, we found that ARNTL2 was enriched in most cell cycle-related terms (Figure 7C). For GSEA results based on KEGG and Reactome, ARNTL2 was enriched in cell cycle, Focal adhesion, PI3K-Akt signaling, Adaptive immune system, and innate immune system pathways (Figure 7D-F). For the GSVA results, we found that ARNTL2 was positively correlated with most oncogenic pathways, such as PI3K AKT MTOR signaling, Glycolysis, Hypoxia, Inflammatory response, and Interferon gamma response pathways (Figure 8). As we have proved that ARNTL2 was highly expressed in LUAD, and high expression of ARNTL2 indicated bad survival of LUAD patients. These results indicated that ARNTL2-regulated cell cycle-related and immune-related pathways may contribute to the poor survival of patients with tumors.

\section{Immune cell infiltration analysis}

To understand the role of ARNTL2 in TIME of LUAD, we performed the correlation analysis between ARNTL2 expression and stromal and immune scores calculated by R package "ESTIMATE". Results indicated that ARNTL2 was positively correlated with stromal score, ESTIMATE score, and immune score and negatively correlated with tumor purity in most tumor types (Figure 9A) and LUAD (Figure 9B-E).

By analyzing the association between ARNTL2 expression and immune cell infiltration using the ImmuCellAl database, we found that ARNTL2 expression was positively associated with tumor associated macrophages (TAMs) and Tregs infiltration in pan-cancer (Figure 10A) and LUAD (Figure 10B). According to the results of TIMER2, we observed that ARNTL2 was positively associated with TAMs and cancer associated fibroblasts (CAFs) infiltration (Figure 11).

We also conducted a correlation analysis between immunosuppressive genes and ARNTL2. We found that ARNTL2 expression was positively correlated with most immunosuppressive genes in LUAD (Figure 12A). In addition, we also found that ARNTL2 expression was positively associated with immune checkpoints in LUAD, such as CD274 (PD-L1), CTLA4, LAG3, PDCD1 (PD-1), and TIGIT (Figure 12B). We further proved that ARNTL2 was closely associated with immunomodulatory genes, including MHC genes (Figure 13A), chemokines (Figure 13B) and chemokine receptors (Figure 13C).

\section{Drug resistance analysis}


Additionally, we analyzed the correlation between ARNTL2 and IC50 of 192 drugs. Among the 192 anticancer drugs, ARNTL2 expression was positively correlated with IC50 of 114 anti-cancer drugs, such as SB505124, Doramapimod, Nutlin-3a (-), Sabutoclax, AZD5991, PF-4708671, Elephantin, PRIMA-1MET, Sorafenib, Vorinostat, and MK-2206 (Figure 14, Supplementary Table 1).

\section{Discussion}

LUAD is one of the most common cancers and is a fatal factor in the health of people globally [14-16]. Recent studies have shown that the remodeling of TIME by cancer cells acts as a critical role in the progression of LUAD, weakening the response of LUAD patients to treatment and leading to worse

survival status ${ }^{[17-20]}$. Thus, the identification of essential genes that could affect TIME is urgently needed.

ARNTL2 is one of the PAS superfamily proteins. Several studies have reported the oncogenic role of ARNTL2 in tumors. However, the function of ARNTL2 in LUAD, especially in regulating TIME, remains unknown. In our study, we first assessed the expression of ARNTL2 and found the elevated ARNTL2 expression in 26 of the 33 cancer types, including LUAD. We also found that the methylation and CNA of ARNTL2 have important implications for ARNTL2 mRNA expression in LUAD. To determine the prognostic role of ARNTL2, we conducted the UniCox and Kaplan-Meier survival analysis in TCGA cohort. Results of the UniCox indicated that ARNTL2 acts as a risk factor for OS, DSS, DFI, and PFI in patients with LUAD. Kaplan-Meier OS analysis revealed that an elevated ARNTL2 expression indicated worse survival of patients with LGG, LUAD, MESO, PAAD, UCEC, and UVM. These results indicate that ARNTL2 is a prognostic marker in most tumor types, including LUAD.

GSEA and GSVA results predicted the immune regulatory function of ARNTL2 in LUAD. We also found that the infiltration levels of TAMs, CAFs, and Tregs were closely associated with ARNTL2 expression in LUAD and pan-cancer. Through the correlation analysis between ARNTL2 expression and immunosuppressive genes and immune checkpoints, we found that ARNTL2 expression was positively correlated with most immunosuppressive genes in LUAD, such as TGFBR1, IL10, IL10RB, CTLA4, PDCD1, CD274, LAG3, and TIGIT. In addition, we also found that ARNTL2 expression was positively associated with immunomodulatory genes, including MHC genes, chemokines and chemokine receptors.

Additionally, we also performed the correlation analysis between ARNTL2 and IC50 of 192 anti-cancer drugs. We found that patients with high ARNTL2 expression might be resistant to most anti-cancer drugs, such as SB505124, Doramapimod, Nutlin-3a (-), Sabutoclax, AZD5991, PF-4708671, Elephantin, PRIMA$1 \mathrm{MET}$, Sorafenib, Vorinostat, and MK-2206.

In conclusion, our findings revealed that ARNTL2 is a potential oncogene and a prognostic marker in LUAD. High ARNTL2 expression may contribute to the immunosuppressive microenvironment in LUAD. Targeting ARNTL2 may be a potential method for LUAD therapy. 


\section{Abbreviations}

ACC: Adrenocortical carcinoma; BLCA: Bladder Urothelial Carcinoma; LUAD: Breast invasive carcinoma; CESC: Cervical squamous cell carcinoma and endocervical adenocarcinoma; $\mathrm{CHOL}$ : Cholangiocarcinoma; COAD: Colon adenocarcinoma; DLBC: Lymphoid Neoplasm Diffuse Large B-cell Lymphoma; ESCA: Esophageal carcinoma; GBM: Glioblastoma multiforme; HNSC: Head and Neck squamous cell carcinoma; $\mathrm{KICH}$ : Kidney Chromophobe; KIRC: Kidney renal clear cell carcinoma; KIRP: Kidney renal papillary cell carcinoma; LAML: Acute Myeloid Leukemia; LGG: Lower Grade Glioma; LIHC: Liver hepatocellular carcinoma; LUAD: Lung adenocarcinoma; LUSC: Lung squamous cell carcinoma; MESO: Mesothelioma; OV: Ovarian serous cystadenocarcinoma; PAAD: Pancreatic adenocarcinoma; PCPG: Pheochromocytoma and Paraganglioma; PRAD: Prostate adenocarcinoma; READ: Rectum adenocarcinoma; SARC: Sarcoma; SKCM: Skin Cutaneous Melanoma; STAD: Stomach adenocarcinoma; TGCT: Testicular Germ Cell Tumor; THCA: Thyroid carcinoma; THYM: Thymoma; UCEC: Uterine Corpus Endometrial Carcinoma; UCS: Uterine Carcinosarcoma; UVM: Uveal Melanoma; GSEA: Gene set enrichment analysis; GSVA: Gene Set Variation Analysis; GDSC: Genomics of Drug Sensitivity in Cancer database; GO: Gene Ontology; KEGG: Kyoto Encyclopedia of Genes and Genomes.

\section{Declarations}

\section{Author Contributions}

GW conceived and designed the study.GW acquired the data and drafted the manuscript. WW critically revised the manuscript. ZY performed the statistical analysis and technical support. QX provided all the funding for this study.All authors contributed to the article and approved the submitted version. They have agreed to be accountable for all aspects of the work in ensuring that questions related to the accuracy or integrity of any part of the work are appropriately investigated and resolved.

\section{Funding statement}

QX provided all the funding for this study.

\section{Corresponding authors}

Correspondence to Gujie Wu or Qun Xue.

\section{Institutional email address}

Gujie Wu's institutional email address is is 2013320130@stmail.ntu.edu.cn.

\section{Data Availability Statement}

Publicly available datasets were used for analysis in this study. These data can be obtained at https://xenabrowser.net/datapages/,https://www.cbioportal.org/ portals.We hereby undertake that all 
data and materials are available.

\section{Ethics approval and consent to participate}

TCGA belong to public databases. The patients involved in the database have obtained ethical approval. Users can download relevant data for free for research and publish relevant articles. Our study is based on open source data, so there are no ethical issues and other conflicts of interest. The Ethics Committee of Affiliated Hospital of Nantong University has granted exemptions from approval for research related to the use of such public databases.

\section{Consent for publication}

All the authors agreed to be published.

\section{Competing interests}

The authors declare no conflicts of interest.

\section{Acknowledgements}

\section{None}

\section{References}

1. Denisenko Tatiana V,Budkevich Inna N,Zhivotovsky Boris,Cell death-based treatment of lung adenocarcinoma.[J] .Cell Death Dis, 2018, 9: 117.

2. Zhang Chaoqi,Zhang Zhen,Zhang Guochao et al. Clinical significance and inflammatory landscapes of a novel recurrence-associated immune signature in early-stage lung adenocarcinoma.[J] .Cancer Lett, 2020, 479: 31-41.

3. Zhang Chaoqi,Zhang Guochao,Sun Nan et al. Comprehensive molecular analyses of a TNF familybased signature with regard to prognosis, immune features, and biomarkers for immunotherapy in lung adenocarcinoma.[J] .EBioMedicine, 2020, 59: 102959.

4. Chen Haiquan,Carrot-Zhang Jian,Zhao Yue et al. Genomic and immune profiling of pre-invasive lung adenocarcinoma.[J] .Nat Commun, 2019, 10: 5472.

5. Kim Nayoung,Kim Hong Kwan,Lee Kyungjong et al. Single-cell RNA sequencing demonstrates the molecular and cellular reprogramming of metastatic lung adenocarcinoma.[J] .Nat Commun, 2020, 11: 2285 .

6. Mittal Suruchi,Brown Nicola J,Holen Ingunn,The breast tumor microenvironment: role in cancer development, progression and response to therapy.[J] .Expert Rev Mol Diagn, 2018, 18: 227-243.

7. He Di,Wang Di,Lu Ping et al. Single-cell RNA sequencing reveals heterogeneous tumor and immune cell populations in early-stage lung adenocarcinomas harboring EGFR mutations.[J] .Oncogene, 2021, 40: 355-368. 
8. Lu Chia-Sing,Shiau Ai-Li,Su Bing-Hua et al. Oct4 promotes M2 macrophage polarization through upregulation of macrophage colony-stimulating factor in lung cancer.[J] .J Hematol Oncol, 2020, 13: 62.

9. Suzuki Jun,Aokage Keiju,Neri Shinya et al. Relationship between podoplanin-expressing cancerassociated fibroblasts and the immune microenvironment of early lung squamous cell carcinoma.[J] .Lung Cancer, 2021, 153: 1-10.

10. Cresswell Gregory M,Wang Bingbing,Kischuk Erin M et al. Folate Receptor Beta Designates Immunosuppressive Tumor-Associated Myeloid Cells That Can Be Reprogrammed with FolateTargeted Drugs.[J] .Cancer Res, 2021, 81: 671-684.

11. Wang Zhifang,Liu Tingting,Xue Wenhua et al. ARNTL2 promotes pancreatic ductal adenocarcinoma progression through TGF/BETA pathway and is regulated by miR-26a-5p.[J].Cell Death Dis, 2020, 11: 692.

12. Lu Min,Huang Liyun,Tang Yinli et al. ARNTL2 knockdown suppressed the invasion and migration of colon carcinoma: decreased SMOC2-EMT expression through inactivation of PI3K/AKT pathway.[J] .Am J Transl Res, 2020, 12: 1293-1308.

13. Wang Song,Ma Xueyou,Ying Yufan et al. Upregulation of ARNTL2 is associated with poor survival and immune infiltration in clear cell renal cell carcinoma.[J] .Cancer Cell Int, 2021, 21: 341.

14. Jiang Neng,Zou Chang,Zhu Ying et al. HIF-1区-regulated miR-1275 maintains stem cell-like phenotypes and promotes the progression of LUAD by simultaneously activating Wnt/ $\beta$-catenin and Notch signaling.[J] .Theranostics, 2020, 10: 2553-2570.

15. Feng Tienan,Zhang Qi,Li Qiang et al. LUAD transcriptomic profile analysis of d-limonene and potential IncRNA chemopreventive target.[J] .Food Funct, 2020, 11: 7255-7265.

16. Li Yin,Gu Jie,Xu Fengkai et al. Molecular characterization, biological function, tumor microenvironment association and clinical significance of m6A regulators in lung adenocarcinoma. [J] .Brief Bioinform, 2021, 22: undefined.

17. Dai Jiawei,Jiang Minlin,He Kan et al. DNA Damage Response and Repair Gene Alterations Increase Tumor Mutational Burden and Promote Poor Prognosis of Advanced Lung Cancer.[J] .Front Oncol, 2021, 11: 708294.

18. Chen Jing,Zhang Kai,Zhi Yingru et al. Tumor-derived exosomal miR-19b-3p facilitates M2 macrophage polarization and exosomal LINC00273 secretion to promote lung adenocarcinoma metastasis via Hippo pathway.[J] .Clin Transl Med, 2021, 11: e478.

19. Zhang Wencheng,Gao Zhouyong,Guan Mingxiu et al. ASF1B Promotes Oncogenesis in Lung Adenocarcinoma and Other Cancer Types.[J] .Front Oncol, 2021, 11: 731547.

20. Zhang Pengfei,He Boxue,Cai Qidong et al. Decreased IL-6 and NK Cells in Early-Stage Lung Adenocarcinoma Presenting as Ground-Glass Opacity.[J] .Front Oncol, 2021, 11: 705888.

\section{Figures}



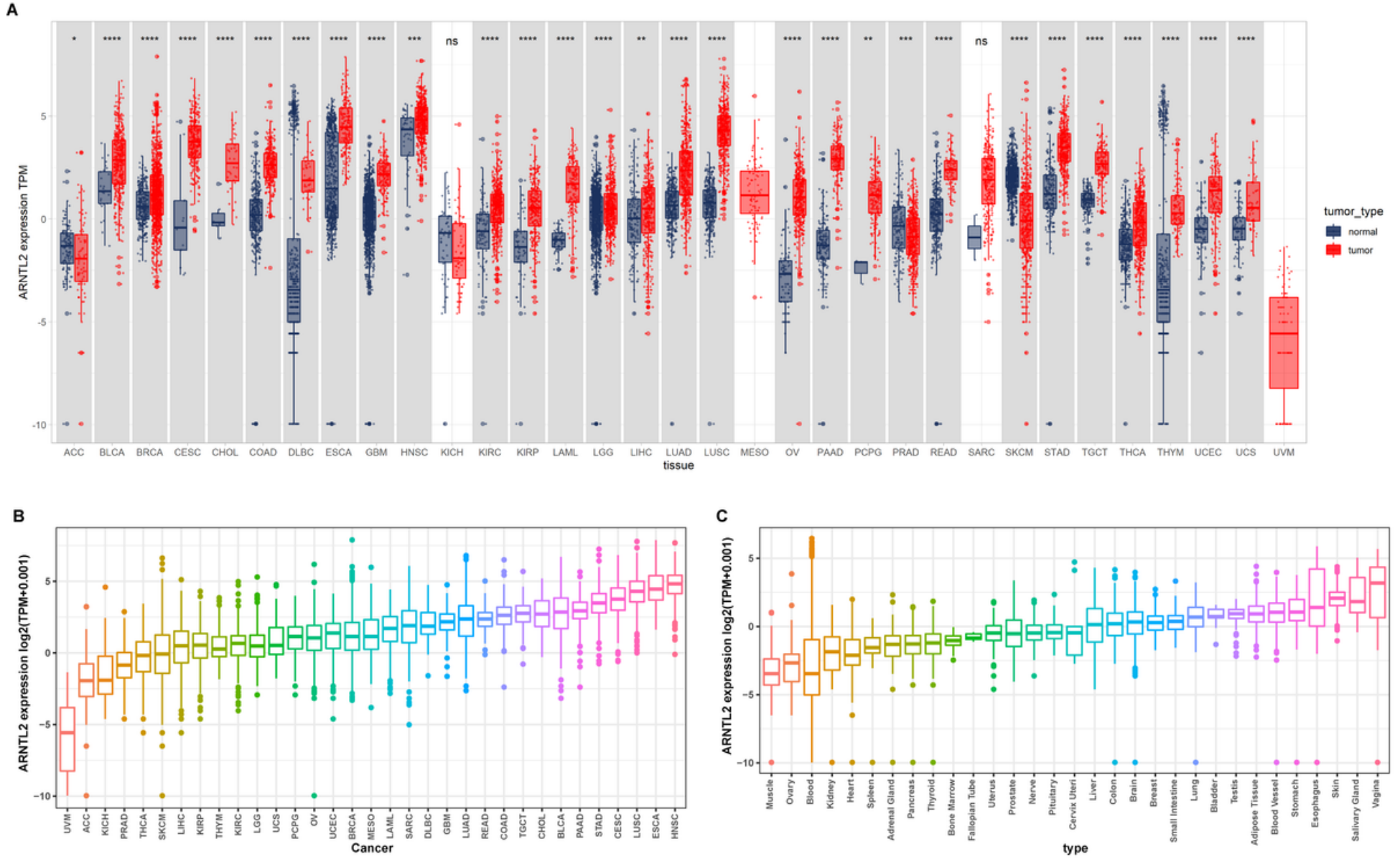

\section{Figure 1}

Expression of ARNTL2 in pan-cancer. (A) pan-cancer expression of ARNTL2. (B) ARNTL2 expression in tumor tissues from TCGA cohort. (C) ARNTL2 expression in normal tissues from GTEx cohort. * $P<0.05$,

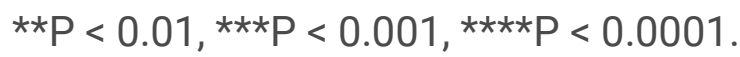



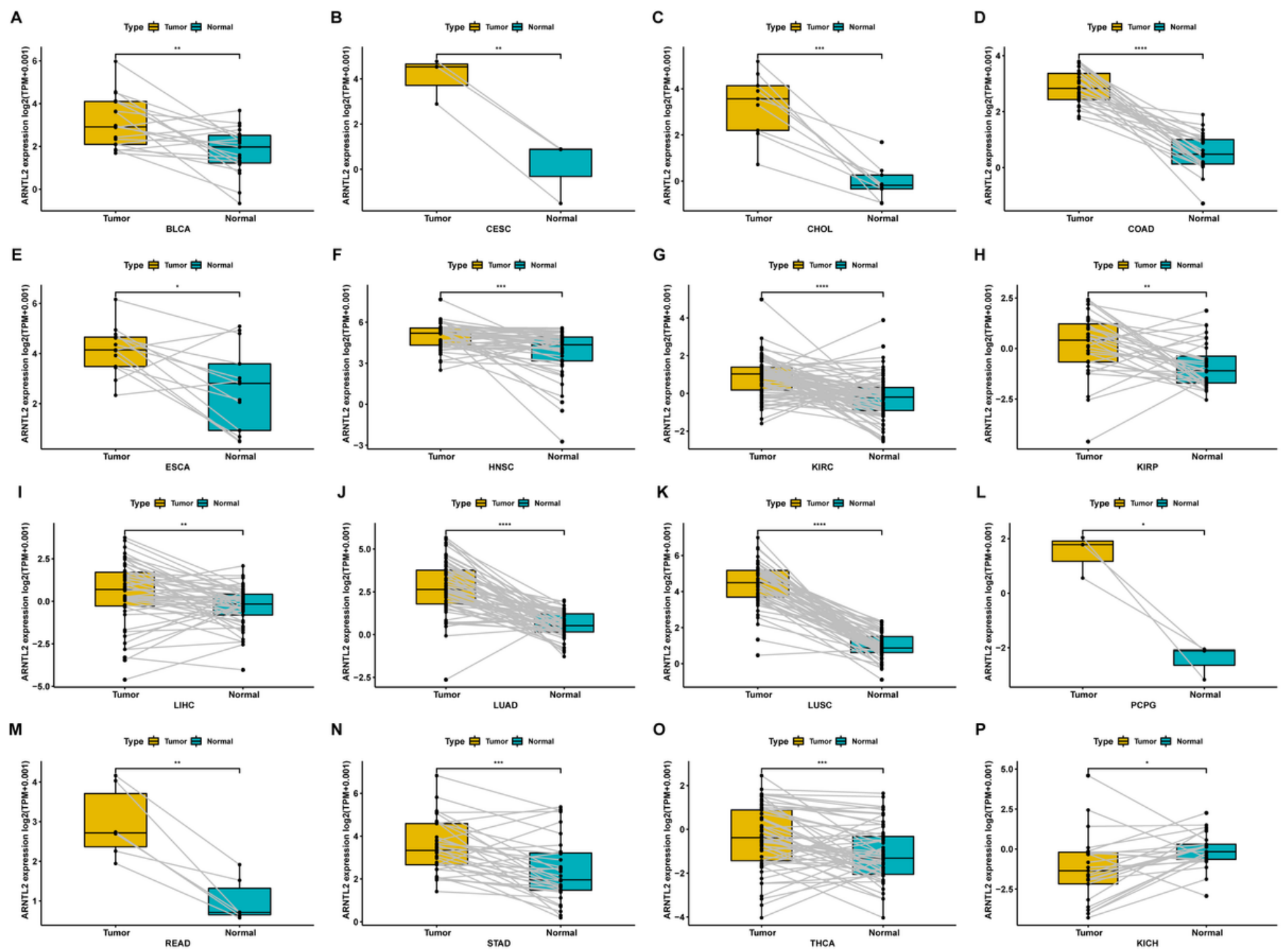

Figure 2

Expression of ARNTL2 in paired tumor and adjacent normal tissues. (A-P) ARNTL2 expression in paired tumor and adjacent normal tissues from TCGA in indicated tumor types. ${ }^{*} \mathrm{P}<0.05,{ }^{*} \mathrm{P}<0.01,{ }^{\star \star *} \mathrm{P}<$ $0.001, \star \star \star \star \mathrm{P}<0.0001$. 
A
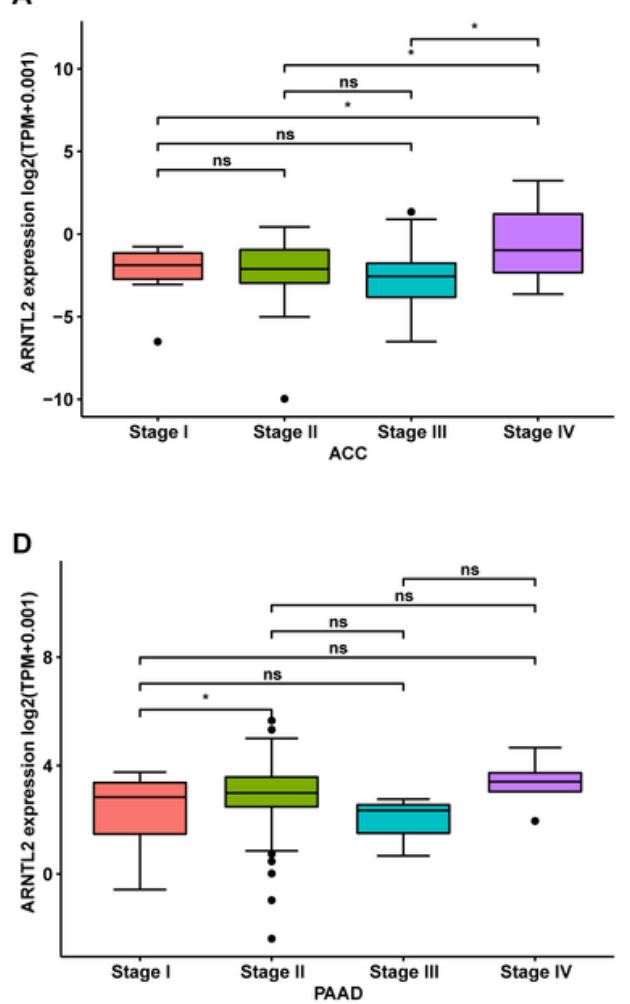

B

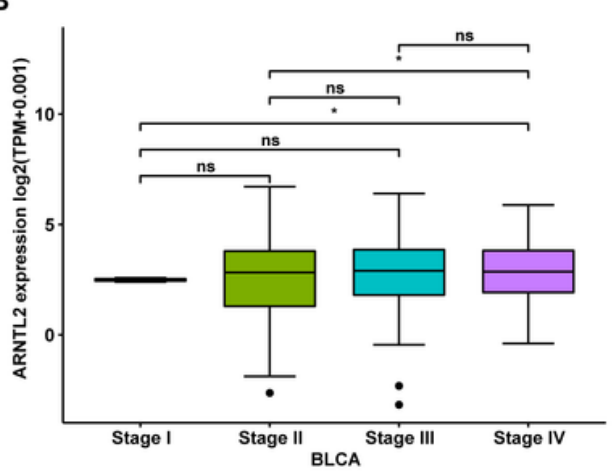

E

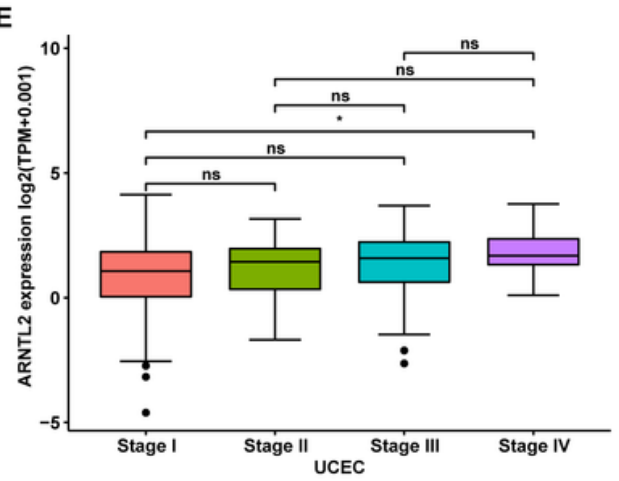

C

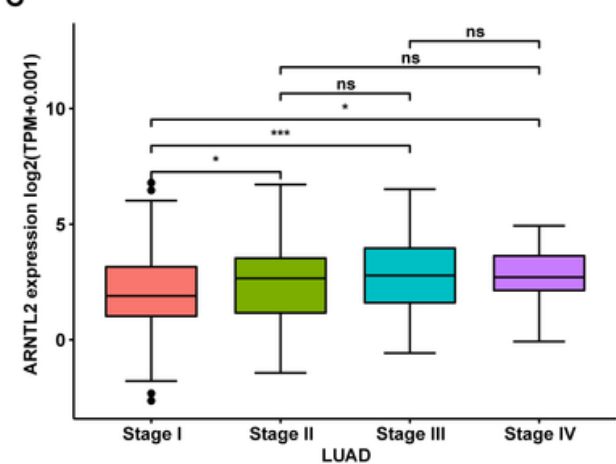

$\mathbf{F}$

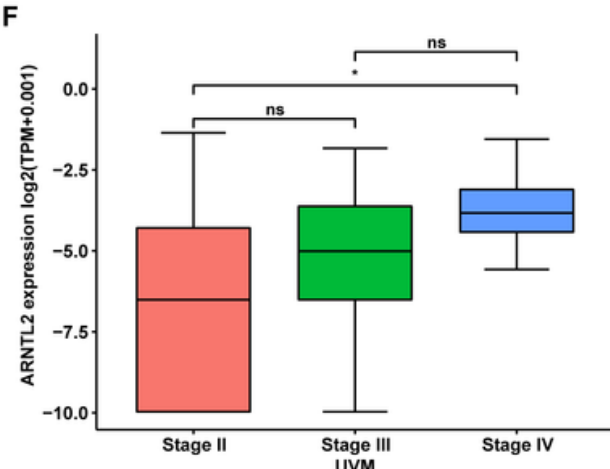

Figure 3

Expression of ARNTL2 in various tumor stages. (A-F) ARNTL2 expression in various tumor stages in indicated tumor types. ${ }^{*} \mathrm{P}<0.05,{ }^{\star * \star} \mathrm{P}<0.001$. 

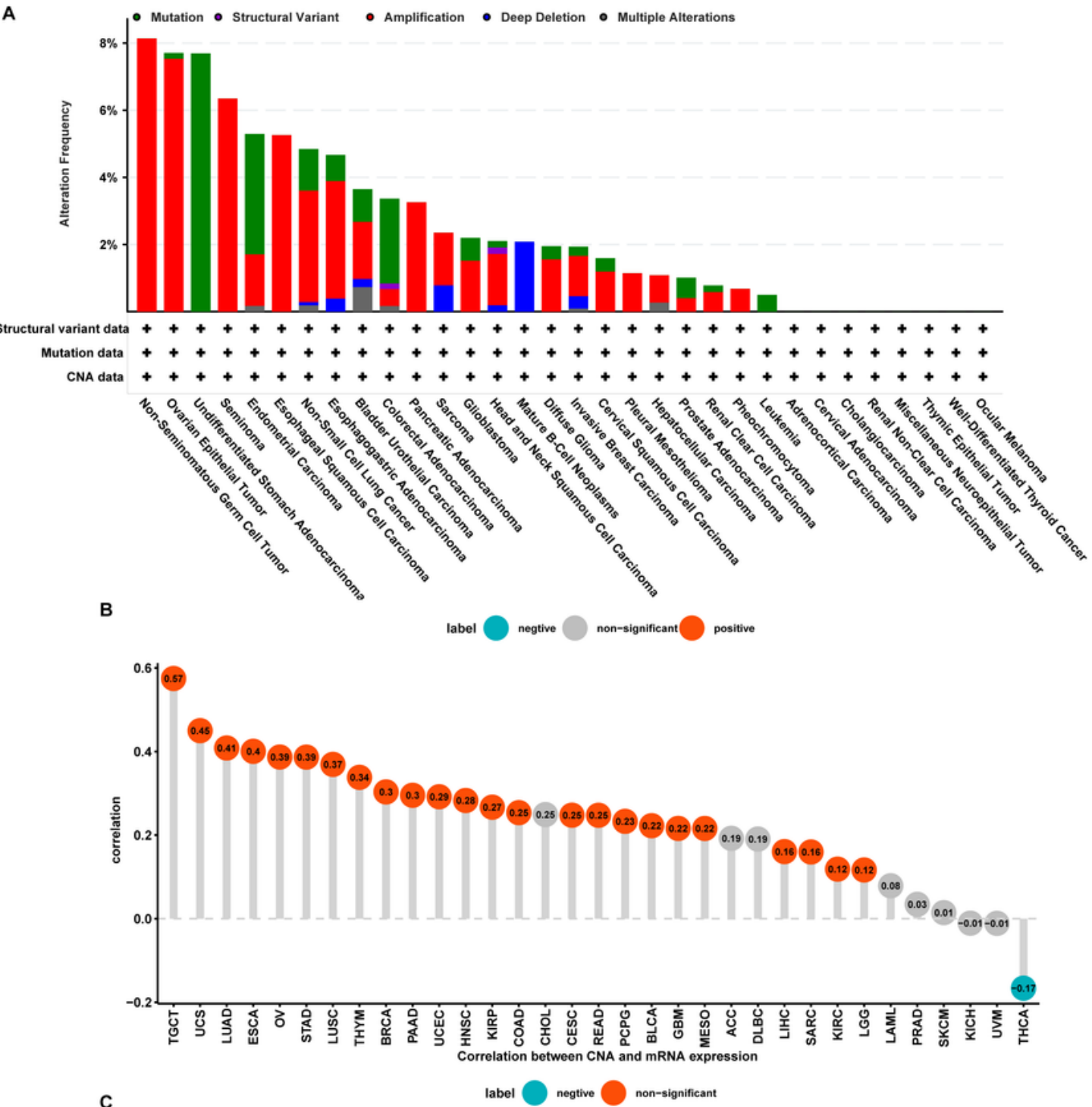

C

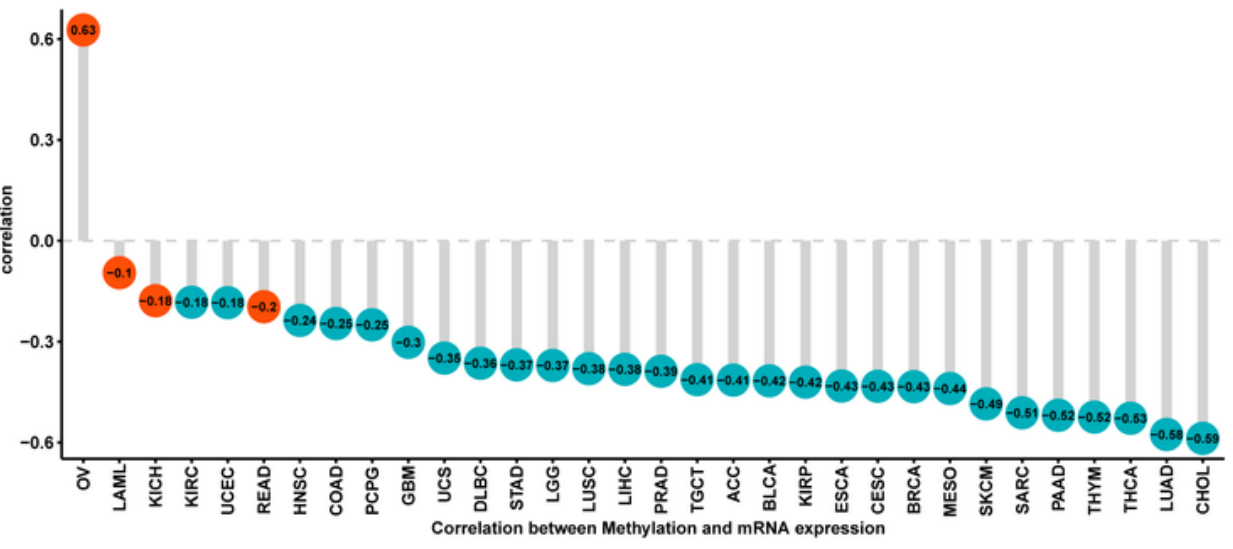

Figure 4

Gene alteration of ARNTL2. (A) The mutation and CNA status of ARNTL2 in TCGA-pan-cancer. (B) The correlation between ARNTL2 expression and CNA. (C) The correlation between ARNTL2 expression and DNA methylation. 


\begin{tabular}{|c|c|c|}
\hline & pvalue & Hazard ratio \\
\hline ACC & 0.039 & $1.245(1.012-1.532)$ \\
\hline BLCA & 0.129 & $1.079(0.978-1.191)$ \\
\hline BRCA & 0.430 & $0.960(0.869-1.062)$ \\
\hline CESC & 0.160 & $1.167(0.941-1.447)$ \\
\hline $\mathrm{CHOL}$ & 0.825 & $1.041(0.727-1.492)$ \\
\hline COAD & 0.571 & $0.935(0.742-1.179)$ \\
\hline DLBC & 0.943 & $1.022(0.558-1.873)$ \\
\hline ESCA & 0.556 & $0.946(0.785-1.139)$ \\
\hline GBM & 0.079 & $1.180(0.981-1.418)$ \\
\hline HNSC & 0.703 & $0.975(0.858-1.109)$ \\
\hline $\mathrm{KICH}$ & 0.032 & $1.547(1.038-2.305)$ \\
\hline KIRC & 0.205 & $1.092(0.953-1.253)$ \\
\hline KIRP & 0.502 & $1.086(0.854-1.382)$ \\
\hline LAML & 0.268 & $1.089(0.937-1.266)$ \\
\hline LGG & $<0.001$ & $1.666(1.432-1.939)$ \\
\hline LIHC & 0.009 & $1.147(1.034-1.271)$ \\
\hline LUAD & $<0.001$ & $1.241(1.125-1.369)$ \\
\hline LUSC & 0.918 & $1.006(0.902-1.122)$ \\
\hline MESO & $<0.001$ & $1.449(1.225-1.714)$ \\
\hline OV & 0.672 & $0.981(0.895-1.074)$ \\
\hline PAAD & $<0.001$ & $1.635(1.336-2.000)$ \\
\hline PCPG & 0.770 & $0.918(0.519-1.626)$ \\
\hline PRAD & 0.612 & $1.131(0.702-1.822)$ \\
\hline READ & 0.027 & $0.548(0.321-0.935)$ \\
\hline SARC & 0.351 & $1.056(0.941-1.185)$ \\
\hline SKCM & 0.326 & $0.971(0.916-1.030)$ \\
\hline STAD & 0.247 & $0.918(0.793-1.062)$ \\
\hline TGCT & 0.719 & $1.218(0.416-3.564)$ \\
\hline THCA & 0.531 & $1.117(0.791-1.577)$ \\
\hline THYM & 0.160 & $1.482(0.856-2.565)$ \\
\hline UCEC & 0.108 & $1.241(0.954-1.615)$ \\
\hline UCS & 0.171 & $1.186(0.929-1.513)$ \\
\hline & 0.002 & $1.362(1.121-1.655)$ \\
\hline
\end{tabular}

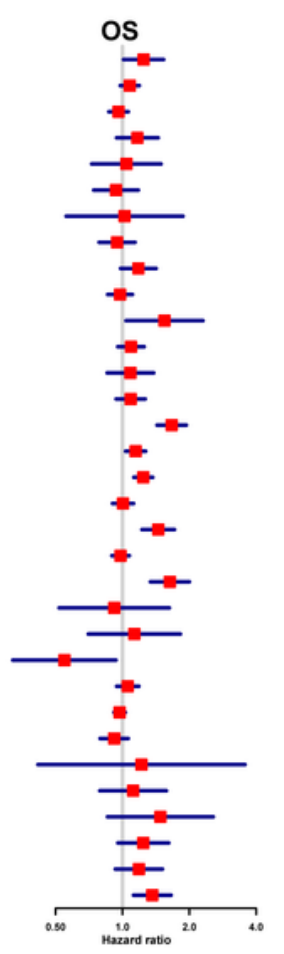

\begin{tabular}{|c|c|}
\hline ACC & 0.086 \\
\hline BLCA & 0.189 \\
\hline BRCA & 0.617 \\
\hline CESC & 0.685 \\
\hline $\mathrm{CHOL}$ & 0.661 \\
\hline COAD & 0.844 \\
\hline DLBC & 0.570 \\
\hline ESCA & 0.686 \\
\hline GBM & 0.077 \\
\hline HNSC & 0.951 \\
\hline $\mathrm{KICH}$ & 0.012 \\
\hline KIRC & 0.164 \\
\hline KIRP & 0.926 \\
\hline LGG & $<0.001$ \\
\hline LIHC & 0.030 \\
\hline LUAD & $<0.001$ \\
\hline LUSC & 0.723 \\
\hline MESO & $<0.001$ \\
\hline OV & 0.289 \\
\hline PAAD & $<0.001$ \\
\hline PCPG & 0.898 \\
\hline PRAD & 0.809 \\
\hline READ & 0.618 \\
\hline SARC & 0.157 \\
\hline SKCM & 0.176 \\
\hline STAD & 0.902 \\
\hline TGCT & 0.979 \\
\hline THCA & 0.85 \\
\hline THYM & 0.11 \\
\hline UCEC & 0.08 \\
\hline UCS & 0.27 \\
\hline UVM & 0.00 \\
\hline
\end{tabular}
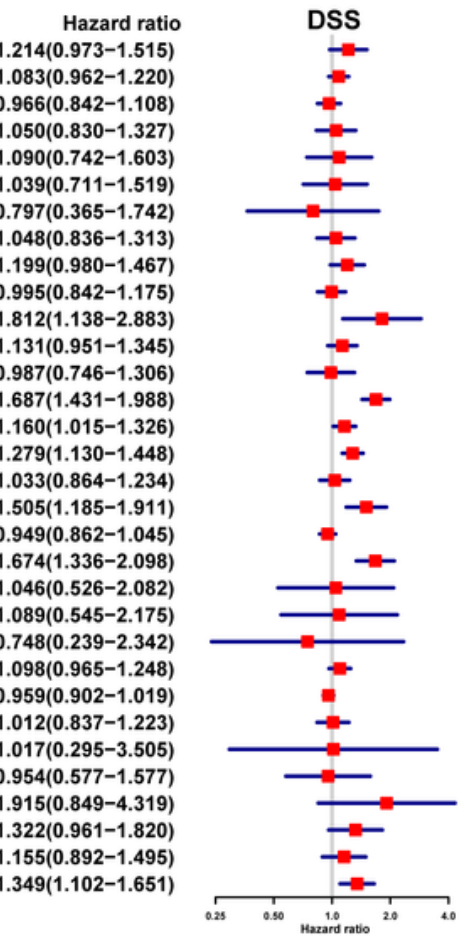

C

pvalue Hazard ratio

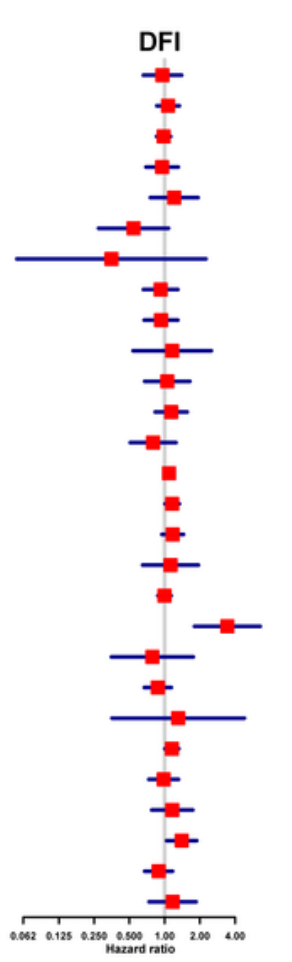

D

\begin{tabular}{|c|c|c|}
\hline & pvalue & Hazard ratio \\
\hline ACC & 0.824 & $0.958(0.658-1.396)$ \\
\hline BLCA & 0.547 & $1.070(0.858-1.335)$ \\
\hline BRCA & 0.776 & $0.980(0.854-1.125)$ \\
\hline CESC & 0.762 & $0.952(0.694-1.307)$ \\
\hline $\mathrm{CHOL}$ & 0.429 & $1.208(0.756-1.929)$ \\
\hline COAD & 0.082 & $0.543(0.273-1.079)$ \\
\hline DLBC & 0.270 & $0.351(0.055-2.255)$ \\
\hline ESCA & 0.648 & $0.924(0.658-1.297)$ \\
\hline HNSC & 0.673 & $0.932(0.670-1.296)$ \\
\hline $\mathrm{KICH}$ & 0.705 & $1.160(0.538-2.502)$ \\
\hline KIRC & 0.818 & $1.054(0.676-1.644)$ \\
\hline KIRP & 0.421 & $1.138(0.831-1.558)$ \\
\hline LGG & 0.323 & $0.797(0.509-1.249)$ \\
\hline LIHC & 0.077 & $1.090(0.991-1.199)$ \\
\hline LUAD & 0.038 & $1.160(1.008-1.336)$ \\
\hline LUSC & 0.141 & $1.172(0.949-1.447)$ \\
\hline MESO & 0.679 & $1.122(0.649-1.941)$ \\
\hline OV & 0.971 & $0.998(0.876-1.137)$ \\
\hline PAAD & $<0.001$ & $3.431(1.799-6.544)$ \\
\hline PCPG & 0.556 & $0.786(0.352-1.755)$ \\
\hline PRAD & 0.321 & $0.875(0.672-1.139)$ \\
\hline READ & 0.688 & $1.306(0.356-4.797)$ \\
\hline SARC & 0.043 & $1.153(1.004-1.323)$ \\
\hline STAD & 0.898 & $0.981(0.733-1.313)$ \\
\hline TGCT & 0.465 & $1.162(0.777-1.738)$ \\
\hline THCA & 0.025 & $1.399(1.042-1.879)$ \\
\hline UCEC & 0.397 & $0.889(0.676-1.168)$ \\
\hline UCS & 0.506 & $1.170(0.737-1.856)$ \\
\hline
\end{tabular}

\begin{tabular}{lr} 
& pvalue \\
ACC & 0.290 \\
BLCA & 0.105 \\
BRCA & 0.894 \\
CESC & 0.847 \\
CHOL & 0.968 \\
COAD & 0.497 \\
DLBC & 0.461 \\
ESCA & 0.850 \\
GBM & 0.120 \\
HNSC & 0.411 \\
KICH & 0.021 \\
KIRC & 0.539 \\
KIRP & 0.595 \\
LGG & $<0.001$ \\
LIHC & 0.028 \\
LUAD & 0.001 \\
LUSC & 0.349 \\
MESO & 0.026 \\
OV & 0.045 \\
PAAD & $<0.001$ \\
PCPG & 0.567 \\
PRAD & 0.621 \\
READ & 0.802 \\
SARC & 0.009 \\
SKCM & 0.442 \\
STAD & 0.217 \\
TGCT & 0.646 \\
THCA & 0.315 \\
THYM & 0.245 \\
UCEC & 0.853 \\
UCS & 0.226 \\
UVM & 0.003 \\
& \\
\hline
\end{tabular}

Hazard ratio 1.094(0.926-1.293) 1.085(0.983-1.198) 0.993(0.892-1.104) 0.981(0.808-1.191) 1.007(0.730-1.388)

0.929(0.751-1.149) $0.817(0.477-1.399)$ $0.983(0.828-1.169)$ 1.160(0.962-1.398)

$0.946(0.828-1.080)$

$1.555(1.070-2.261)$

1.045(0.909-1.201)

0.947(0.775-1.157)

1.362(1.207-1.536)

1.101(1.011-1.199)

1.159(1.060-1.268)

$1.067(0.931-1.223)$

1.225(1.025-1.464)

$0.920(0.848-0.998)$

1.606(1.324-1.950)

0.903(0.637-1.281)

1.041(0.888-1.220)

0.934(0.546-1.597)

1.142(1.033-1.262)

0.981(0.933-1.031)

1.110(0.941-1.309)

$1.088(0.760-1.558)$

1.104(0.910-1.340)

1.233(0.866-1.756)

1.019(0.833-1.247)

1.147(0.918-1.433)

1.286(1.087-1.521)

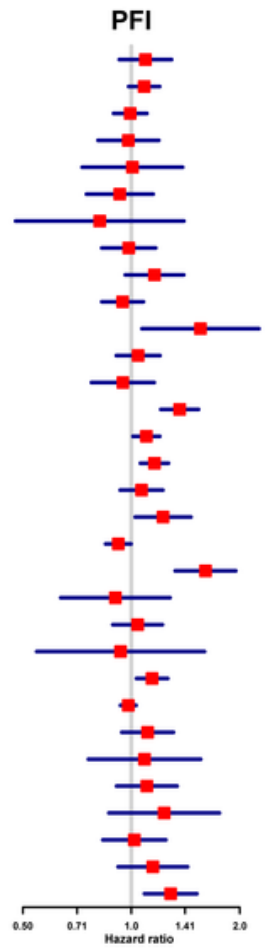

Figure 5

Prognostic value of ARNTL2. (A) The univariate Cox regression OS analysis of ARNTL2 in TCGA pancancer. Red color represents significant results $(p<0.05)$. (B-D) The univariate Cox regression analysis of DSS (B), DFI (C), and PFI (D) in TCGA pan-cancer. 
A

LGG ARNTL2 Survival

Strata - Gene=High expression (263) $\$ Gene=Low expression (255)
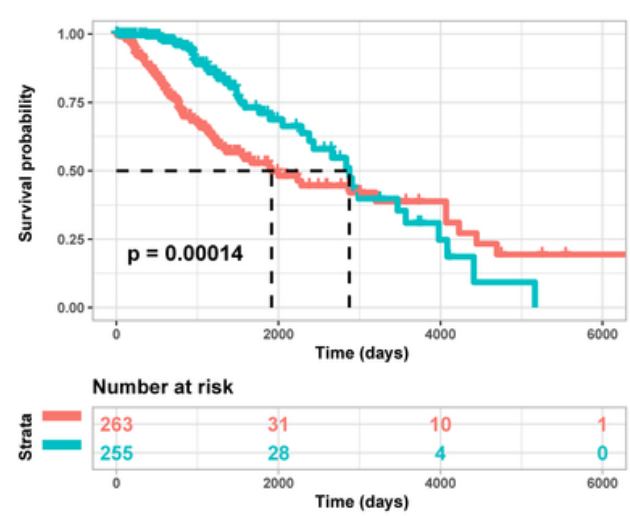

D

PAAD ARNTL2 Survival
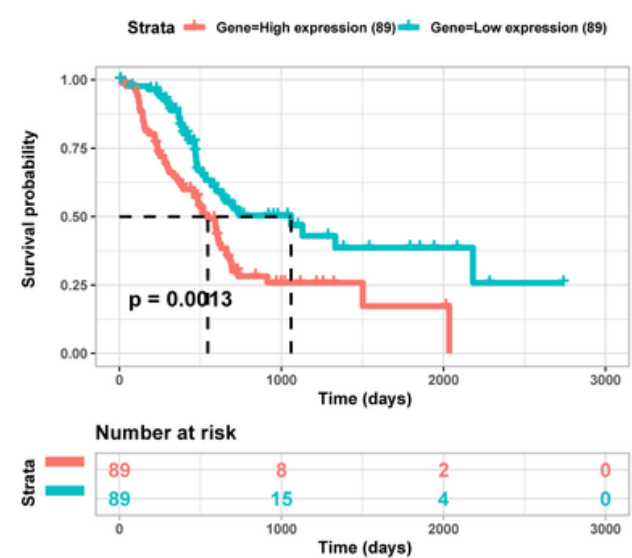

B

LUAD ARNTL2 Survival

Strata $\perp$ Gene=High expression (251) - Gene=Low expression (251)
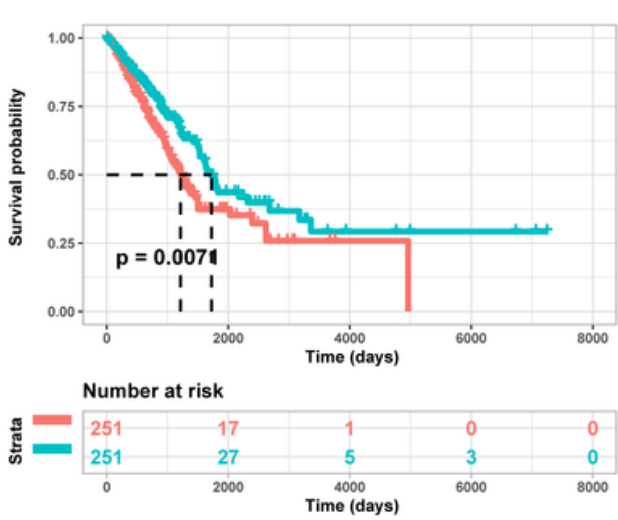

E

UCEC ARNTL2 Survival

Strata $=$ Gone=High exprossion (90) - Gene=Low exprossion (89)
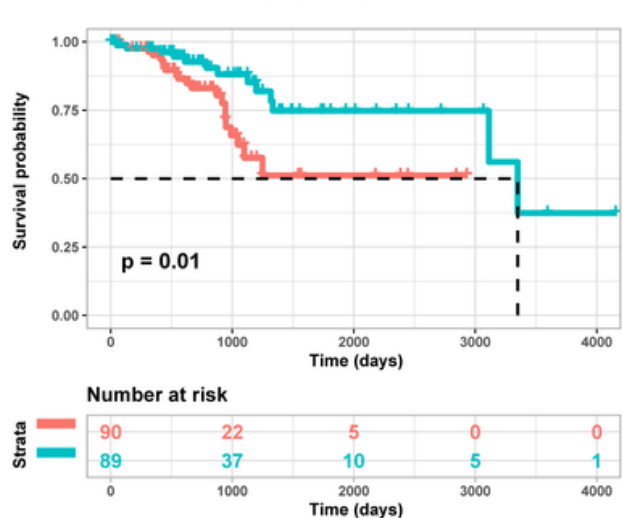

C

MESO ARNTL2 Survival

Strata $\perp$ Gene=High expression (43) $\perp$ Gene=Low expression (42)
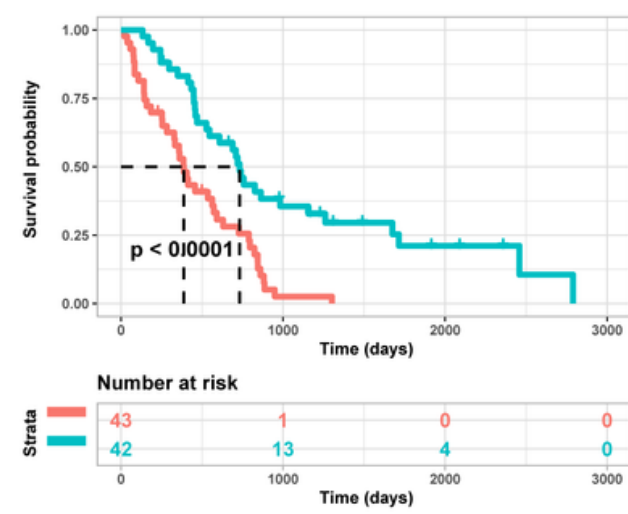

F

UVM ARNTL2 Survival

Strata $=$ Geno=High expression (44) $\perp$ Gene=Low oxprossion (35)

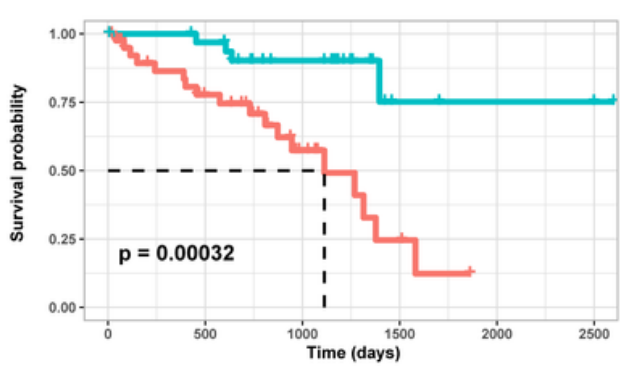

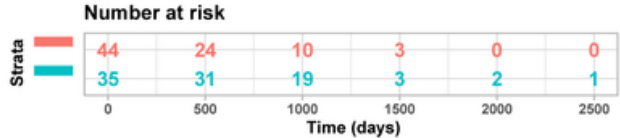

Figure 6

Kaplan-Meier OS analysis of ARNTL2 (A-F) Kaplan-Meier OS analysis of ARNTL2 in TCGA pan-cancer in indicated tumor types. 
A

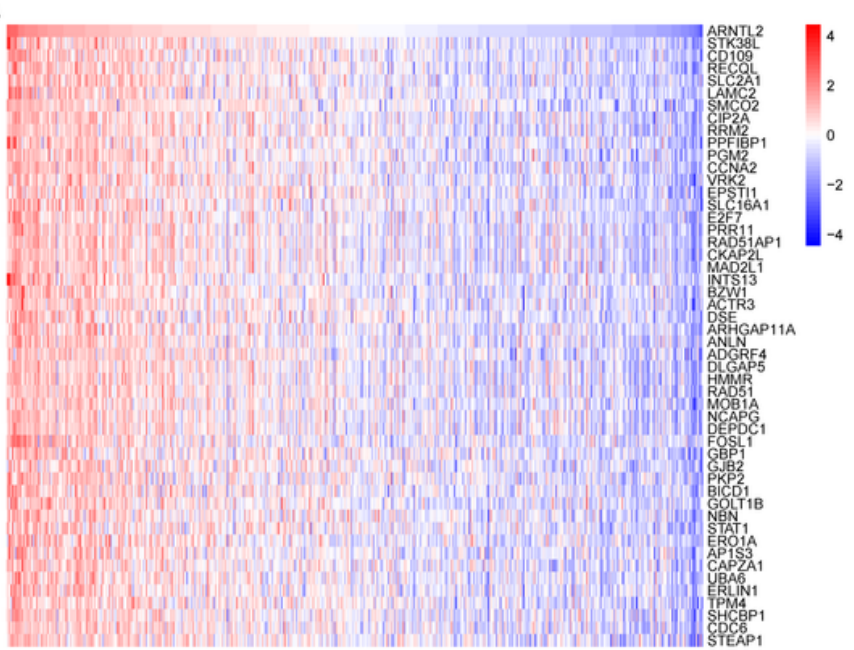

B

C

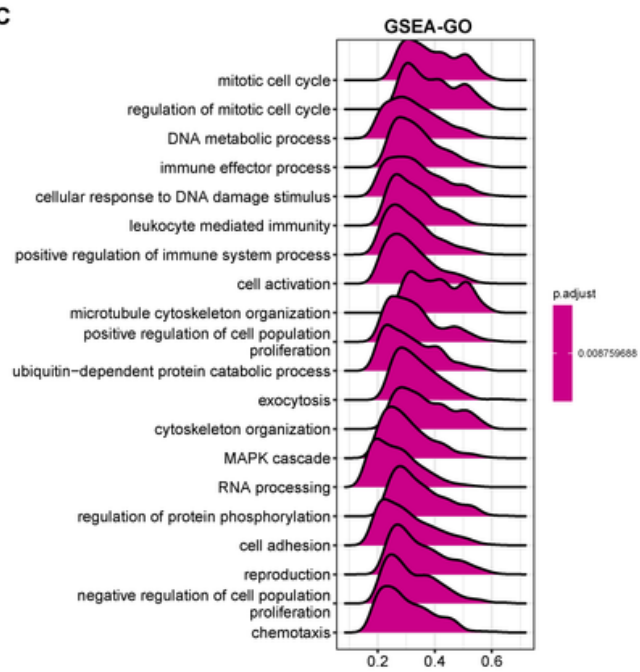

D

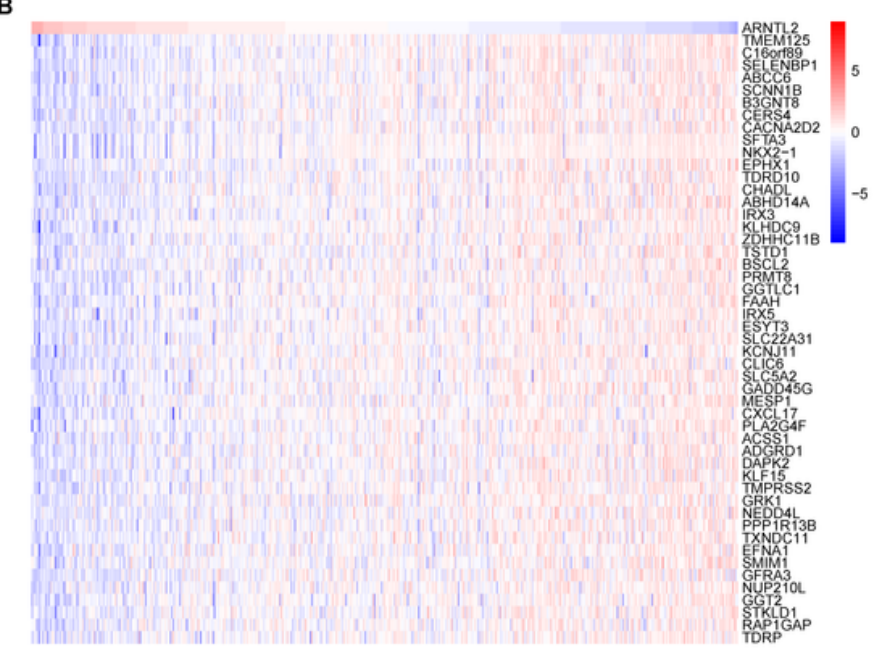

E

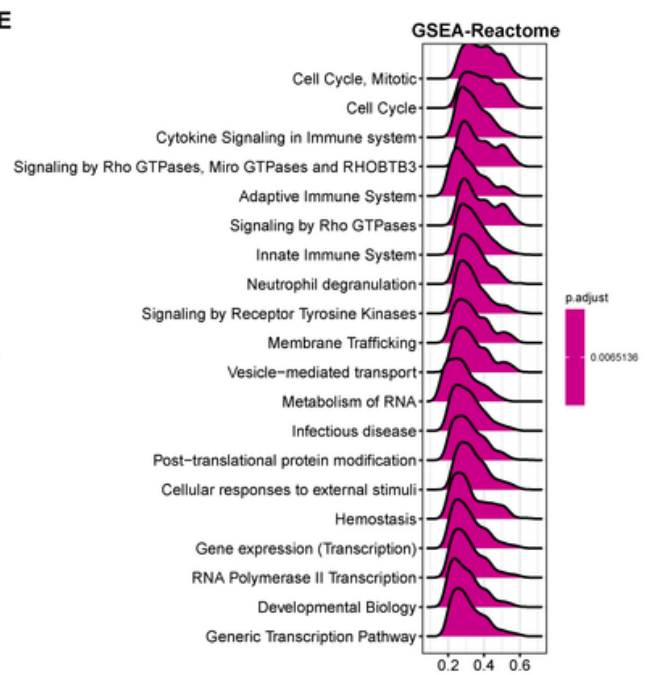

Figure 7

GSEA of ARNTL2. (A) The heatmap of top50 genes positively correlated with ARNTL2 expression. (B) The heatmap of top50 genes negatively correlated with ARNTL2 expression. (C-E) The top 20 GSEA results based on GO (C), KEGG (D), and Reactome (E) database were showed in LUAD. 
PI3K AKT MTOR SIGNALING MTORC1 SIGNALING APICAL SURFACE MITOTIC SPINDLE G2M CHECKPOINT MYC TARGETS V1 GLYCOLYSIS E2F TARGETS HYPOXIA COMPLEMENT TNFA SIGNALING VIA NFKB APOPTOSIS -

INFLAMMATORY RESPONSE INTERFERON GAMMA RESPONSE EPITHELIAL MESENCHYMAL TRANSITION KRAS SIGNALING UP IL6 JAK STAT3 SIGNALING INTERFERON ALPHA RESPONSE ALLOGRAFT REJECTION IL2 STAT5 SIGNALING UNFOLDED PROTEIN RESPONSE ANGIOGENESIS PROTEIN SECRETION TGF BETA SIGNALING APICAL JUNCTION SPERMATOGENESIS UV RESPONSE UP UV RESPONSE DN REACTIVE OXYGEN SPECIES PATHWAY DNA REPAIR -

ANDROGEN RESPONSE MYC TARGETS V2 ESTROGEN RESPONSE LATE COAGULATION WNT BETA CATENIN SIGNALING P53 PATHWAY HEDGEHOG SIGNALING ESTROGEN RESPONSE EARLY CHOLESTEROL HOMEOSTASIS NOTCH SIGNALING -

XENOBIOTIC METABOLISM ADIPOGENESIS HEME METABOLISM OXIDATIVE PHOSPHORYLATION PANCREAS BETA CELLS PEROXISOME MYOGENESIS FATTY ACID METABOLISM KRAS SIGNALING DN BILE ACID METABOLISM -

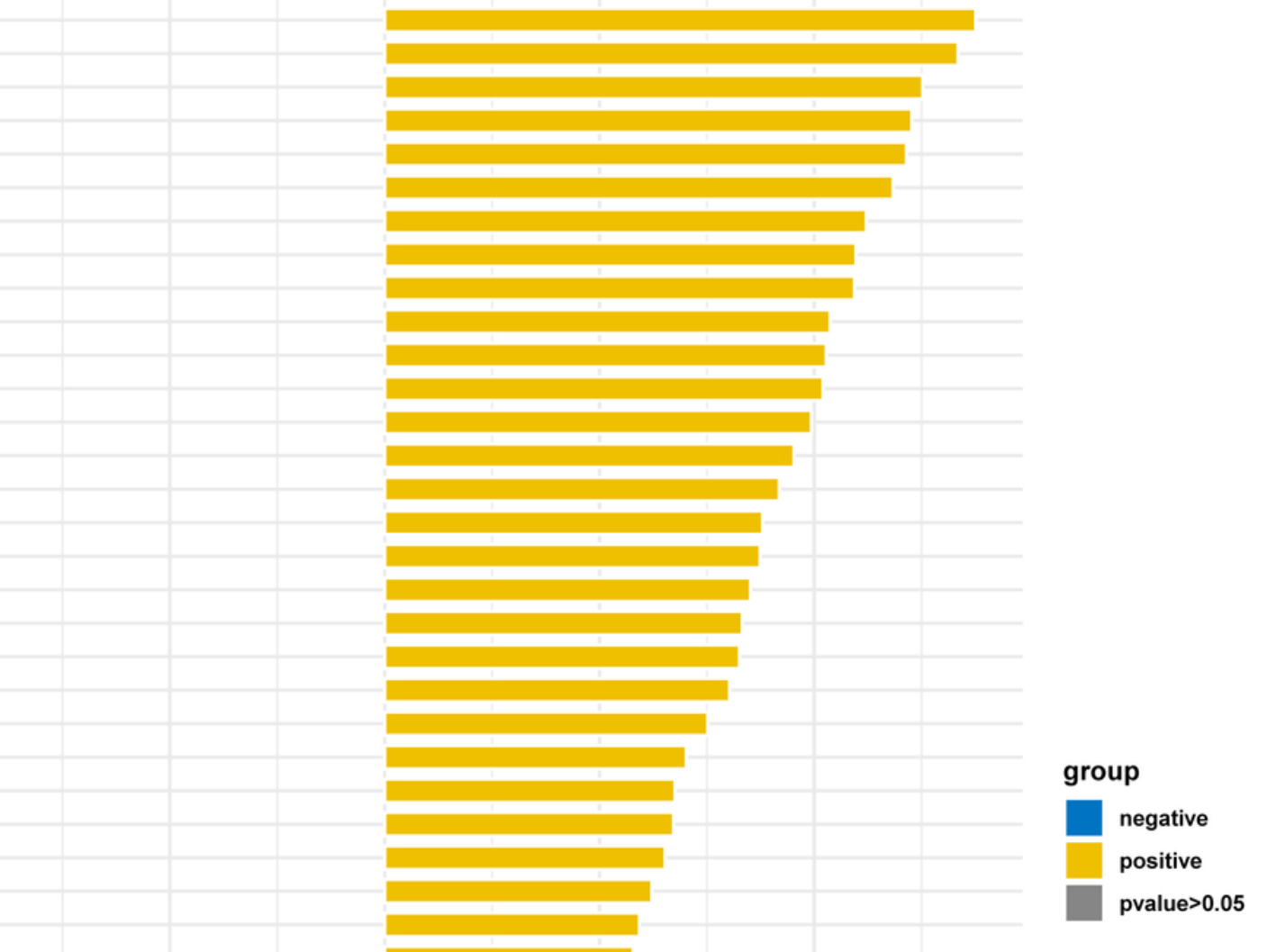


A
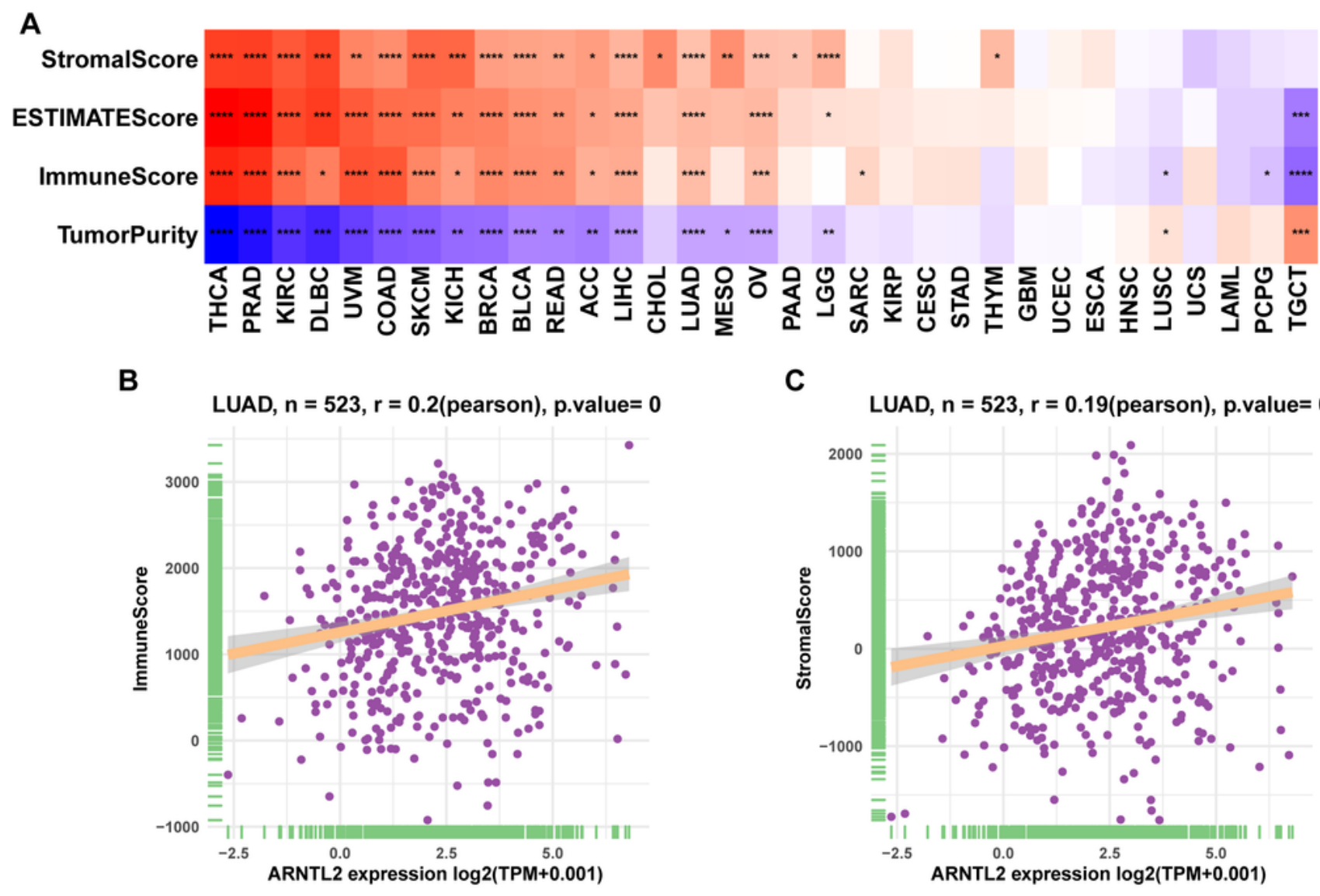

correlation

B

D LUAD, $n=523, r=0.21$ (pearson), p.value $=0$

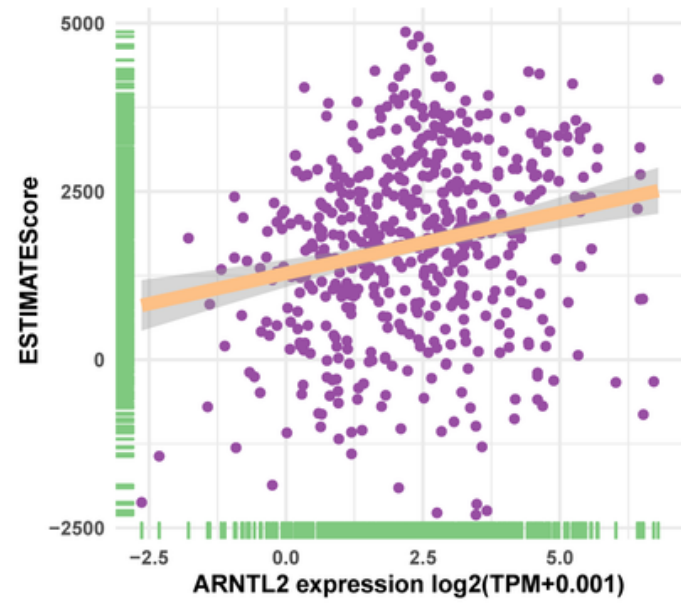

C LUAD, $n=523, r=0.19$ (pearson), p.value $=0$

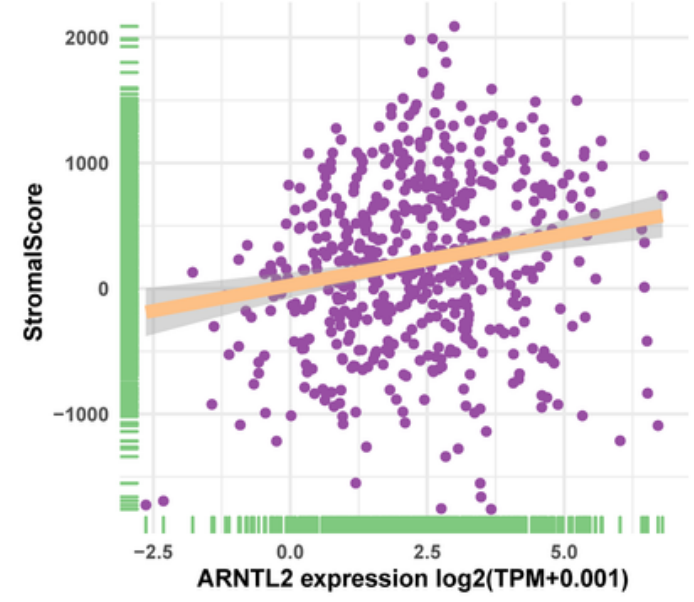

E E LUAD, $n=523, r=-0.22$ (pearson), p.value $=0$

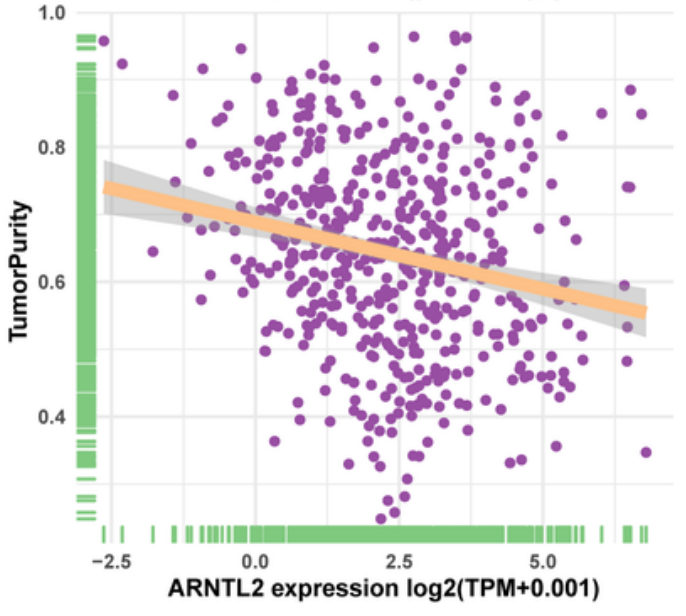

Figure 9

Tumor microenvironment analysis of ARNTL2. (A) Heatmap represents the correlation between ARNTL2 expression and TME scores in pan-cancer. (B-E) The correlation between ARNTL2 expression and immune score (B), stromal score (C), ESTIMATE score (D), and tumor purity score (E). ${ }^{*} P<0.05,{ }^{*} \mathrm{P}<$

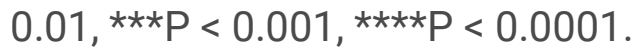




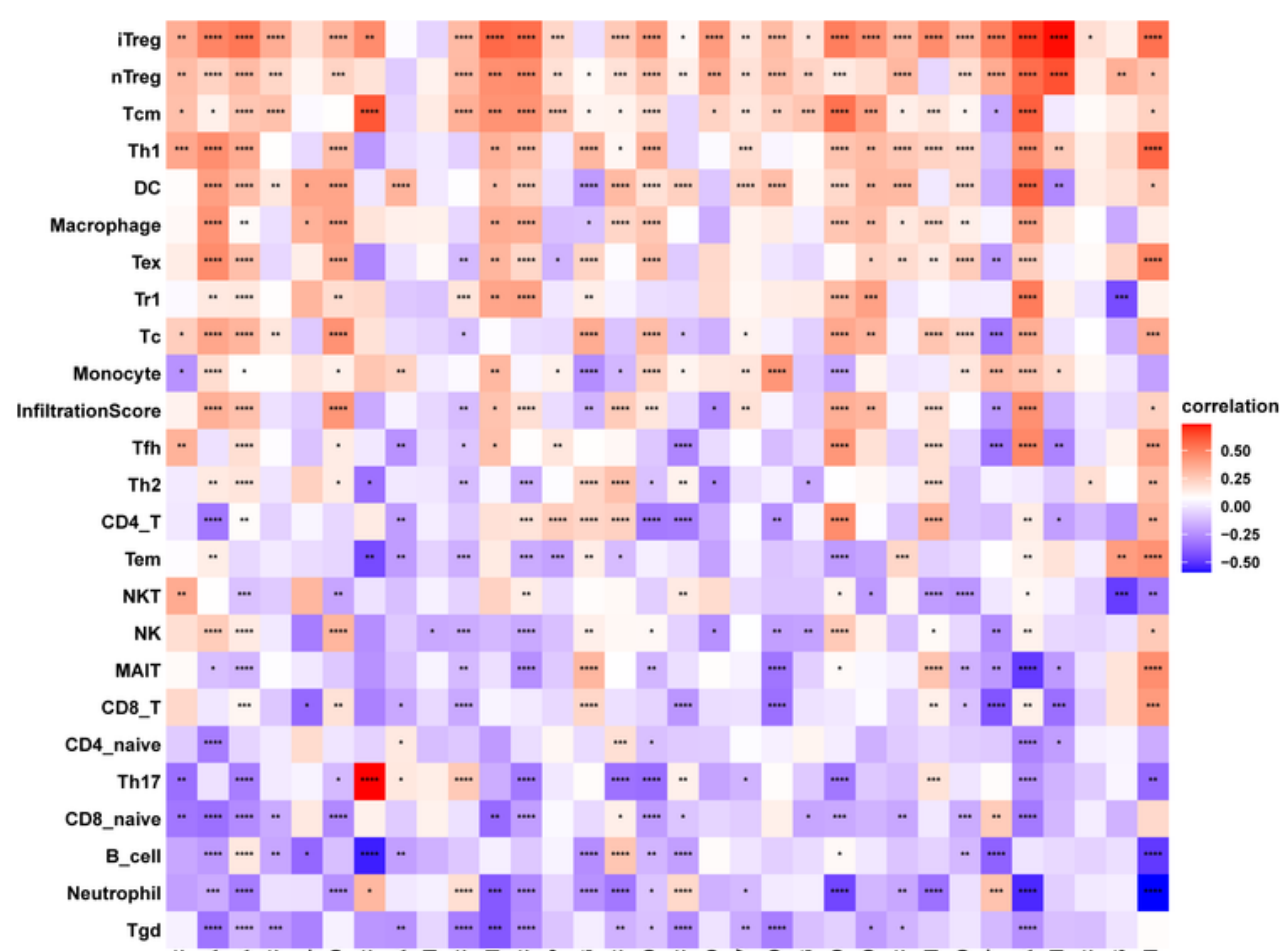

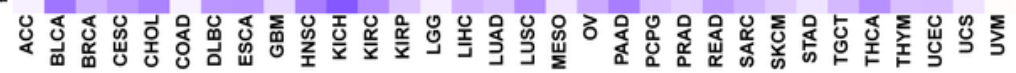

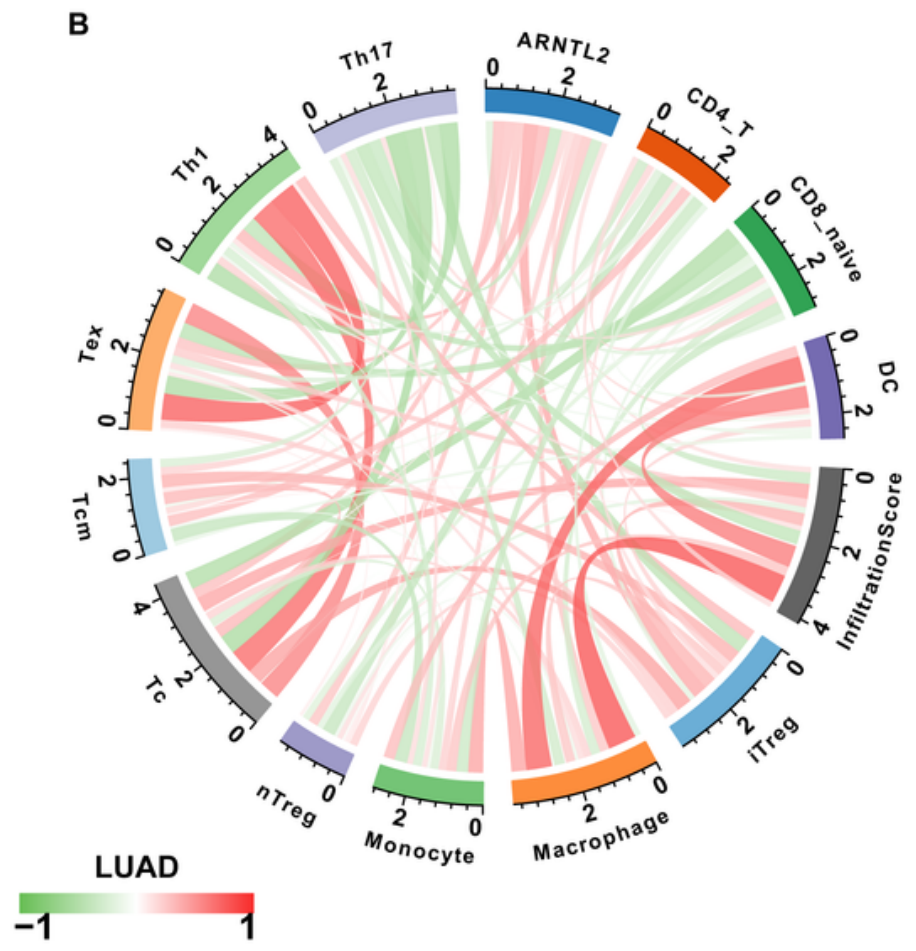

\section{Figure 10}

Immune infiltration analysis based on data from ImmuCellAI database. (A) The relationship between ARNTL2 expression and infiltration levels of 24 immune cells in pan-cancer. (B) The correlation between ARNTL2 expression and immune cells infiltration in LUAD. Red lines represent positive correlation, green lines represent negative correlation, and the darker the color, the stronger the correlation. ${ }^{*} \mathrm{P}<0.05, \star \star \mathrm{P}<$ $0.01,{ }^{\star} * \star \mathrm{P}<0.001, * \star \star \star \mathrm{P}<0.0001$. 


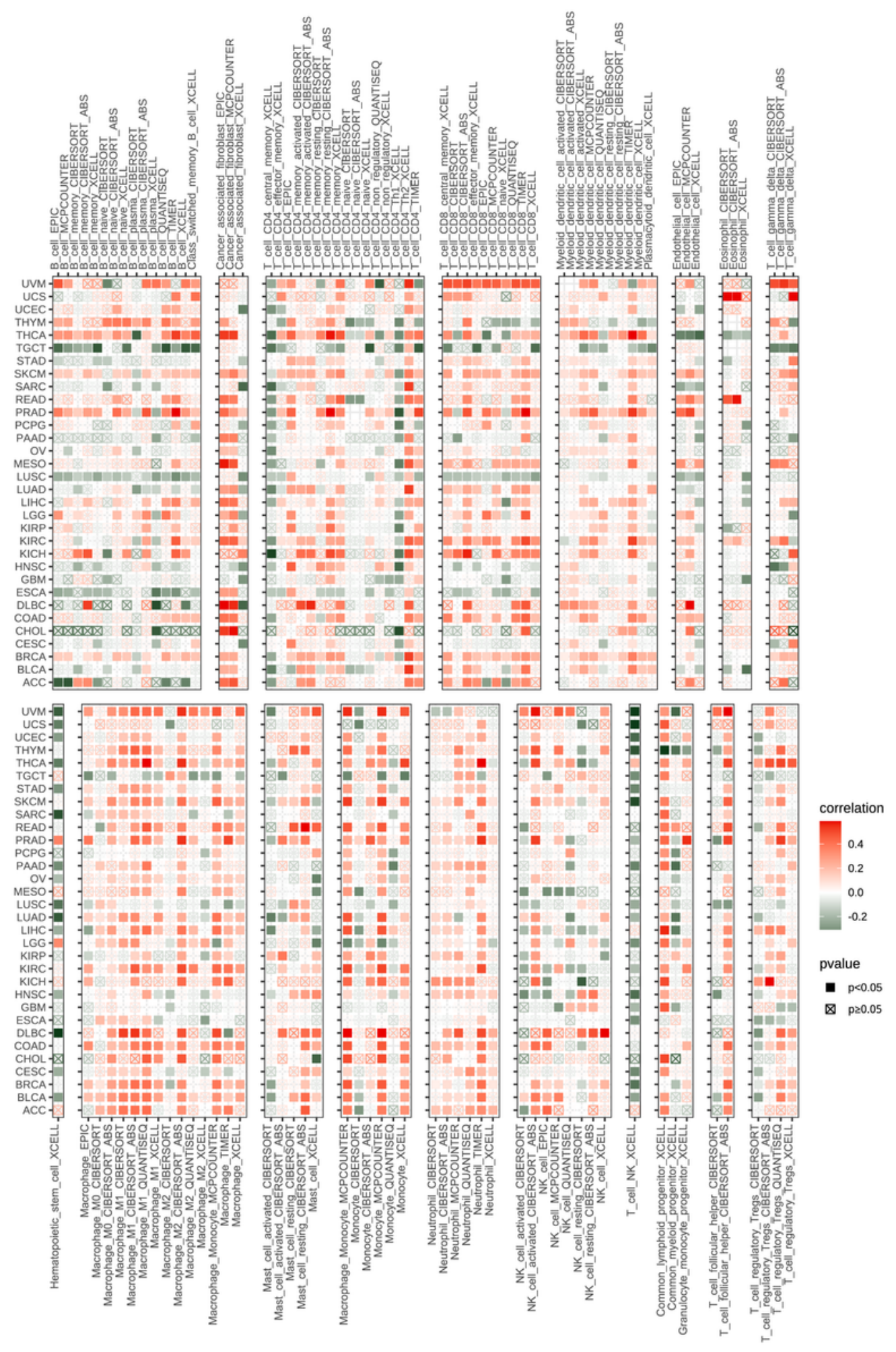

Figure 11

Immune infiltration analysis based on data from TIMER2 database. The relationship between ARNTL2 expression and infiltration levels of immune cells in pan-cancer. Red represents positive correlation, green represents negative correlation, and the darker the color, the stronger the correlation. 
A

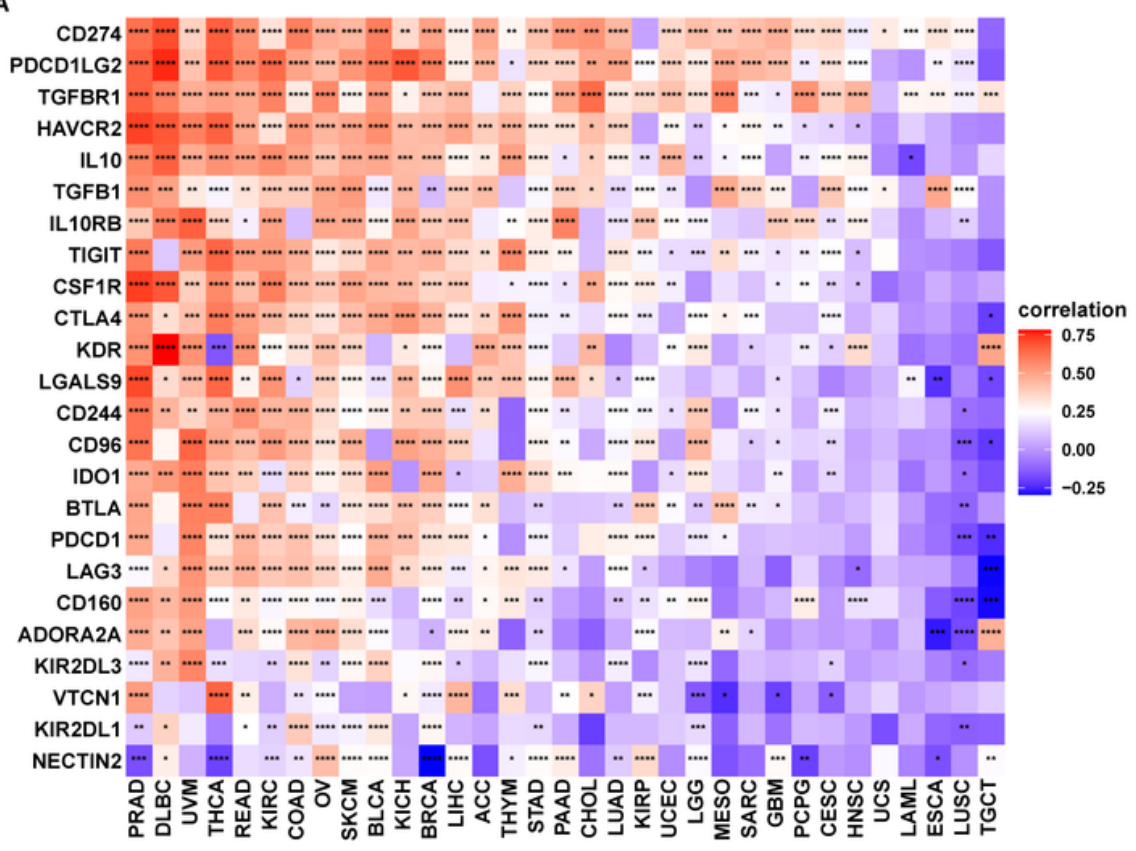

B

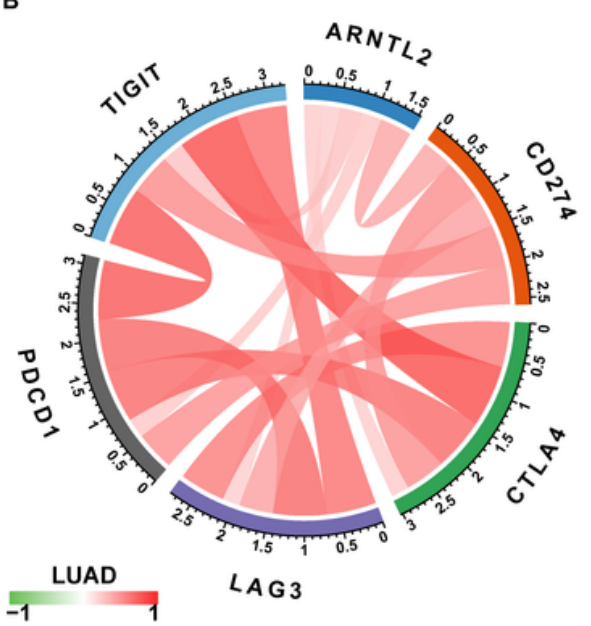

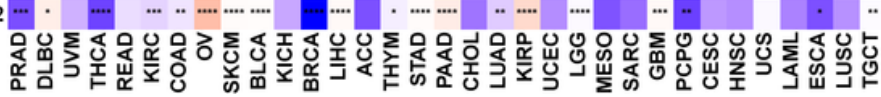

Figure 12

The correlation between ARNTL2 expression and immunosuppressive genes. (A) The correlation between ARNTL2 expression and immunosuppressive genes in pan-cancer. Red color represents positive correlation and blue color represents negative correlation. The darker the color, the stronger the correlation. (B) The correlation between ARNTL2 expression and immune checkpoints in LUAD. ${ }^{*}<<0.05$, $\star \star P<0.01, * \star \star P P 0.001, * \star \star * P<0.0001$. 
A

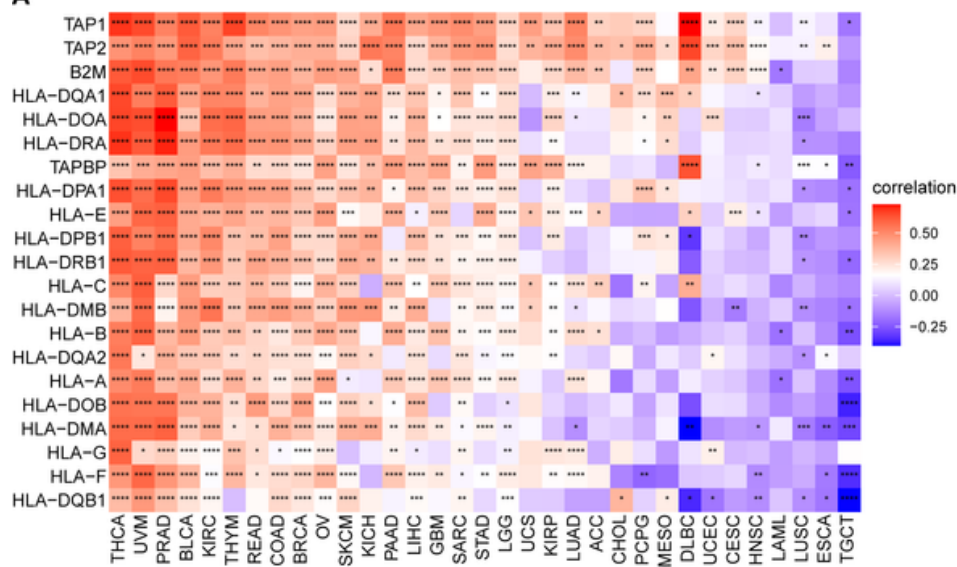

C

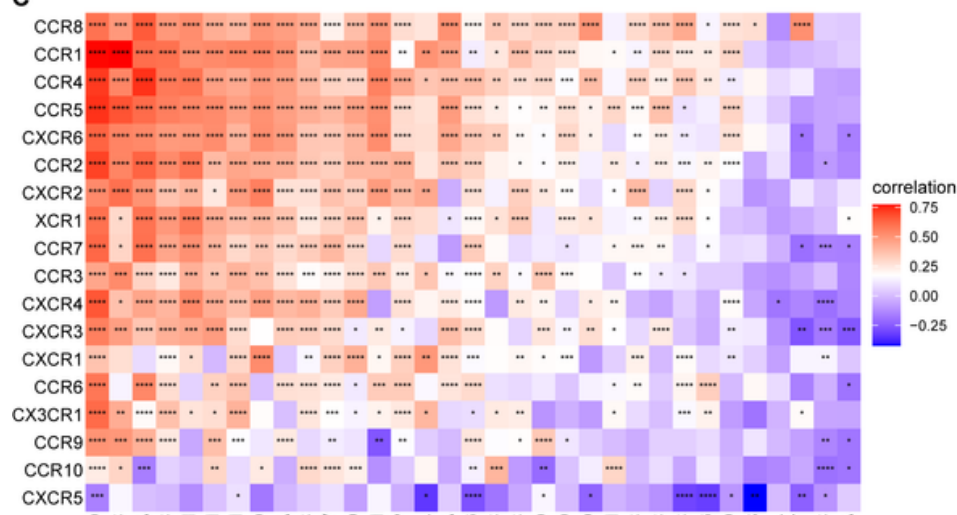

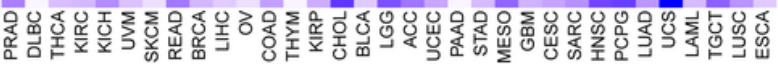

B

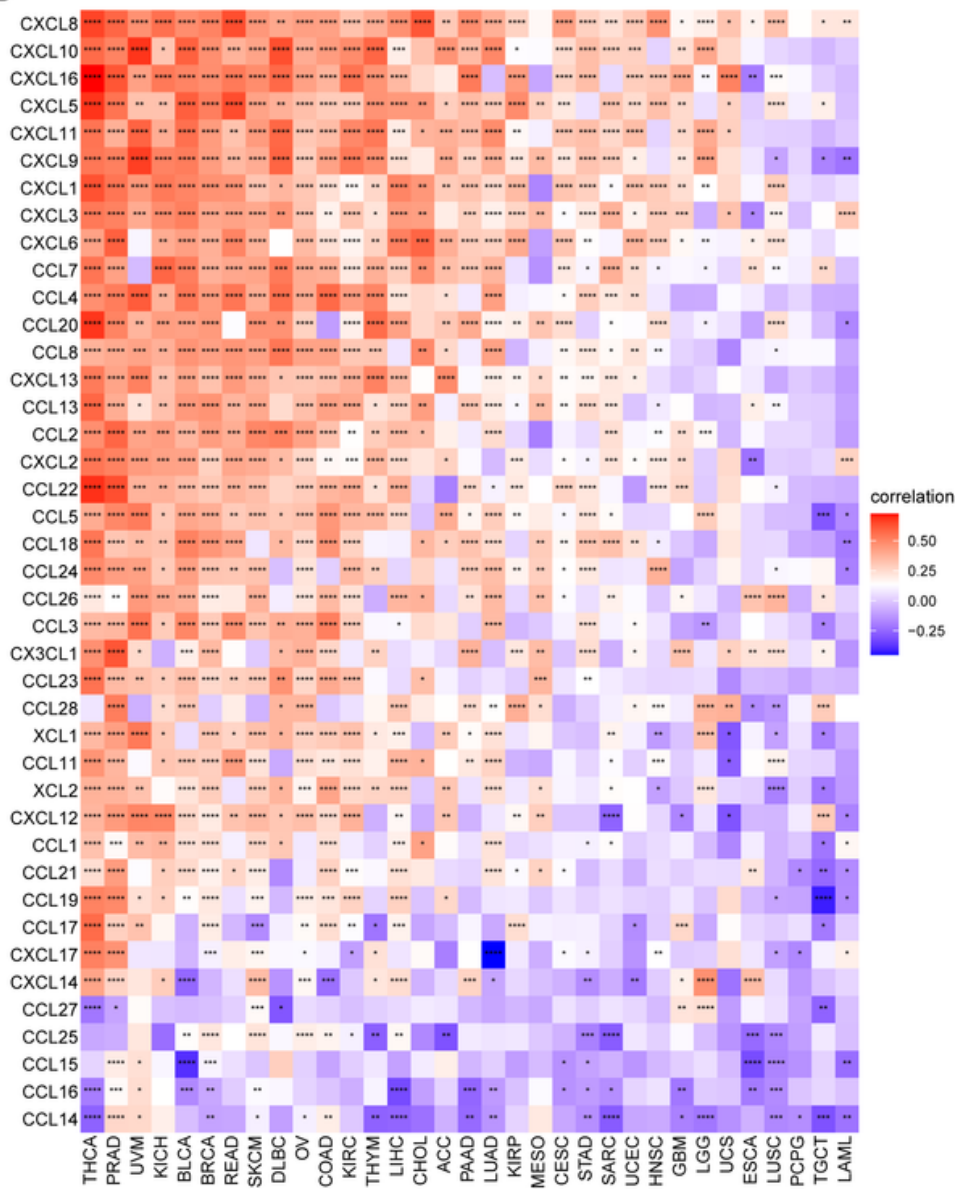

Figure 13

Correlation between ARNTL2 expression and immunomodulatory genes. (A-C) The correlation between ARNTL2 expression and MHC genes (A), chemokines (B) and chemokine receptors (C). ${ }^{*} P<0.05, * \star P<$ $0.01,{ }^{\star * \star} \mathrm{P}<0.001, \star \star \star \star * P<0.0001$. 

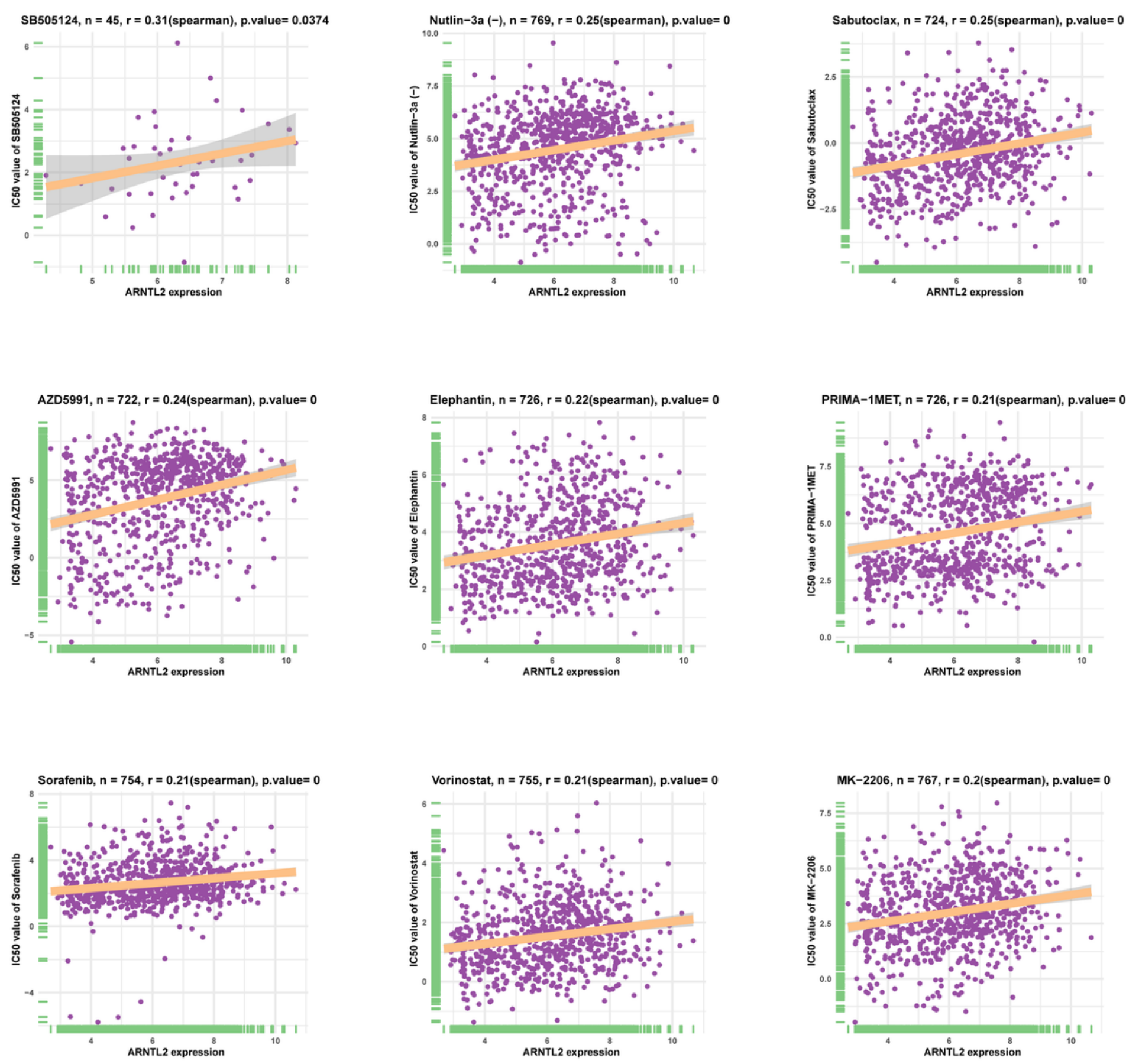

\section{Figure 14}

Drug resistance analysis of ARNTL2 The correlation between ARNTL2 expression and IC50 values of indicated anti-cancer drugs.

\section{Supplementary Files}

This is a list of supplementary files associated with this preprint. Click to download. 